\title{
A state-of-the-art review on magnetorheological elastomer devices
}

\author{
Yancheng $\mathrm{Li}^{1}$, Jianchun $\mathrm{Li}^{1}$, Weihua $\mathrm{Li}^{2}$ and Haiping $\mathrm{Du}^{3}$ \\ 1. Centre for Built Infrastructure Research, School of Civil and Environmental Engineering, Faculty of Engineering and \\ Information Technology, University of Technology Sydney, NSW 2007, Australia \\ 2. School of Mechanical, Materials and Mechatronic Engineering, University of Wollongong, Wollongong, NSW 2522, Australia \\ 3. School of Electrical, Computer and Telecommunications Engineering, University of Wollongong, NSW 2522, Australia
}

\begin{abstract}
:
During last decades, magnetorheological (MR) elastomer has attracted significant amount of attention for its enormous potentials in engineering applications. Being a solid counterpart of MR fluids, MR elastomers exhibit unique field-dependent material property when being exposed to magnetic field, in meanwhile overcoming major issues faced in magnetorheological fluids, e.g. deposition of iron particles, sealing problem and environmental contamination. Such advantages offer great potentials for designing intelligent devices to be used in various engineering fields, especially for vibration reduction and isolation. This paper presents a state-of-the-art review on the recent progress of MR elastomer technology, with special emphasis on research and development of MR elastomer devices and their applications. To keep the integrity of the knowledge, the review includes a brief introduction of the MR elastomer materials and follows by discussion of critical issues in designing magnetorheological elastomer devices, i.e. operation modes, coil placements and principle fundamentals. A comprehensive review has been presented research and development of MR elastomer devices, including vibration absorbers, vibration isolators, base isolators, sensing devices and so on. Summary of research on modeling mechanical behavior for both material and devices are presented. Finally, challenges and potentials facing magnetorheological elastomer technology are discussed and suggestions have been made based on authors' knowledge and experience.
\end{abstract}

Keywords: magnetorheological elastomer, operation modes, vibration absorber, vibration isolator, base isolator, sensing.

\section{Introduction}

Magnetorheological (MR) elastomer, belonging to MR material family, is a composite material with magnetic-sensitive particles suspended or arranged within non-magnetic elastomer matrix [1]. With presence of magnetic field, it exhibits MR effect providing fielddependent material property to the material, e.g. controllable modulus and damping. The material reclaims its original property after the magnetic field is removed. Physical status of the material can be tuned between soft elastomer and semi-solid, depending on the external magnetic field applied to the material [2]. Compared with another member in MR material family - MR fluids, MR elastomers overcome the problems accompanying for applications of MR fluids, such as deposition, sealing issue and environmental contamination, etc., which makes the MR elastomer a favorable candidate for various vibration control application [3].

Although MR effect was firstly discovered by Rabinow in 1940s [4-5], it was not until 1985 the pioneering work on magnetically sensitive elastomer was firstly reported by Rigbi and Jilken [6] with unfulfilled intention for the design of certain medical and measuring 
appliances. A decade later, Shiga et al [7] reported electroviscoelastic effect of a polymer based on silicon elastomer and semiconducting particles under applied electric field. The first comprehensive investigations on MR elastomer was conducted by Jolly and coworkers [8] in 1996. In their work, a quasi-static dipolar model was developed to explain the modulus change inside the MR elastomer. Experimental testing was then conducted to examine the material property of elastomer composite with ferrous particles embedded in a polymer matrix under magnetic field. Since then, research on MR elastomer has attracted increasing attention and become an emerging research topic.

MR elastomers consist of three basic components: polarized magnetic particles, elastomer/rubber matrix and additives (usually silicon oil) [9-10]. The components are mixed together to form a compound with large density magnetic particle randomly dispersed or prearranged in low density matrix. The functionality of the material is based on the magnetic interaction between magnetic particles [9]. Due to the existence of magnetically permeable particles, the composite material exhibits field-dependent material property subjected to external magnetic field where three-dimensional crosslink network is formed between adjacent magnetic particles [11-12]. Such crosslink network tends to retain its original state when external mechanical loading is applied and the tendency is proportional to the magnetic field intensity.

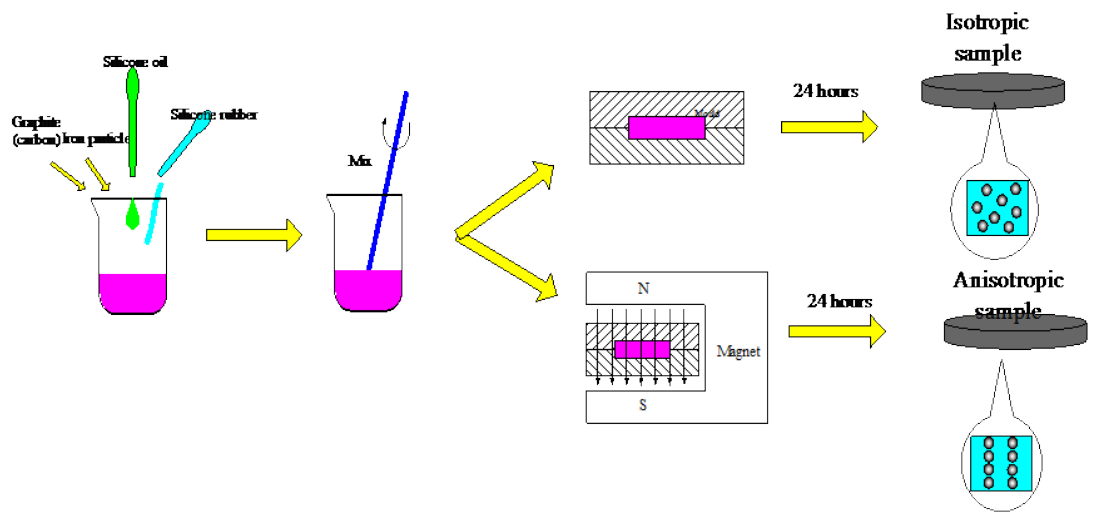

Figure 1. Example of fabrication of both isotropic and anisotropic MR elastomers [17] (note: curing time may vary from case to case)

Generally, high permeability, low remnant magnetization and high saturation magnetization are key features required for the magnetic particles [13]. High permeability of the particles is to easily attract small magnetic leakage fields in the material compound and thus induce maximum possible MR effect [9]. A low remnant magnetization is recommended because the highly remnant particles will stick together after removal of magnetic field due to magnetic residual [14], which therefore makes completely reversible MR effect impossible. The size of the magnetic particles ranges from several micrometers to hundreds of micrometers [11, 14]. The conventional matrixes of MR elastomers are usually natural rubber or silicone rubber, adjusted according to the needs. To prevent particle aggregation as happened to MR fluids as well as to improve the compatibility with polymeric matrix, magnetic particles usually undertake special treatment to remove the moisture from particle surface before curing process [11], which makes the particles to be hydrophobic. Silicone oil is usually used as an additive in MR elastomer material fabrication. When the molecules of silicone oil enter the 
matrix, the gaps between the matrix molecules are increased and the conglutination of molecules is decreased. Apart from increasing the plasticity and fluidity of the matrix, the additives can average the distribution of internal stress in the materials, which makes stable material property for MR elastomer materials [12].

MR elastomers can be categorized into two groups, i.e. isotropic MR elastomers and anisotropic MR elastomers (also termed aligned MR elastomers), which attribute to different ways of curing processes. Firstly, three basic components, namely, silicon rubber, silicon oil and iron particles, are mixed thoroughly into a homogenous mixture. Air bubbles inside the mixture needs to be removed using special treatment, i.e. placed in a vacuum chamber $[9,15]$ or heat treatment [16]. Such treatment maintains the high permeability and uniformity of the material. Isotropic MR elastomer is cured without presence of magnetic field while anisotropic MR elastomer is cured with action of magnetic field, shown in figure 1 [17]. The curing process for anisotropic materials requires strong magnetic field, usually above $0.8 \mathrm{~T}$ [16, 18-19], to form chained structures of magnetic particles in the matrix along the direction of the magnetic field. A constant temperature (usually above $120^{\circ} \mathrm{C}$ ) is also required to maintain the flexibility of the magnetic particle for both isotropic and anisotropic materials $[16,18]$ during curing. Note that some rubber matrix can be cured in room temperature [15, 20]. Prior the curing process, the magnetic particles in the rubber matrix have certain freedom to move around. After curing, the magnetic particles are locked in the matrix [17] and only extra work can force the particles moving away from their original positions. Following curing process, the cured compound undertakes a further chemical process called as vulcanization [16] or polymerization [9]. This is to modify the polymer by forming crosslinks between individual polymer chains and thus creating more durable material. The vulcanizing processes vary from less than 1 hour [16, 18-19] to several days [20], relying on the matrix properties. In general, soft rubber matrix needs more time to stabilize. The vulcanization process makes the material less sticky and having superior mechanical property for engineering applications. In some research, the vulcanization accompanies with application of low magnetic field to further consolidate the chain structure of the magnetic particle [21]. Figure 2 shows the scanning electron micrographs of a freeze-fractured surface of isotropic and anisotropic MR elastomer with 30 \%vol Fe [9]. As observed, Fe particles are randomly dispersed in the isotropic MR elastomer while Fe particles are linked into chain structures in anisotropic MR elastomer.

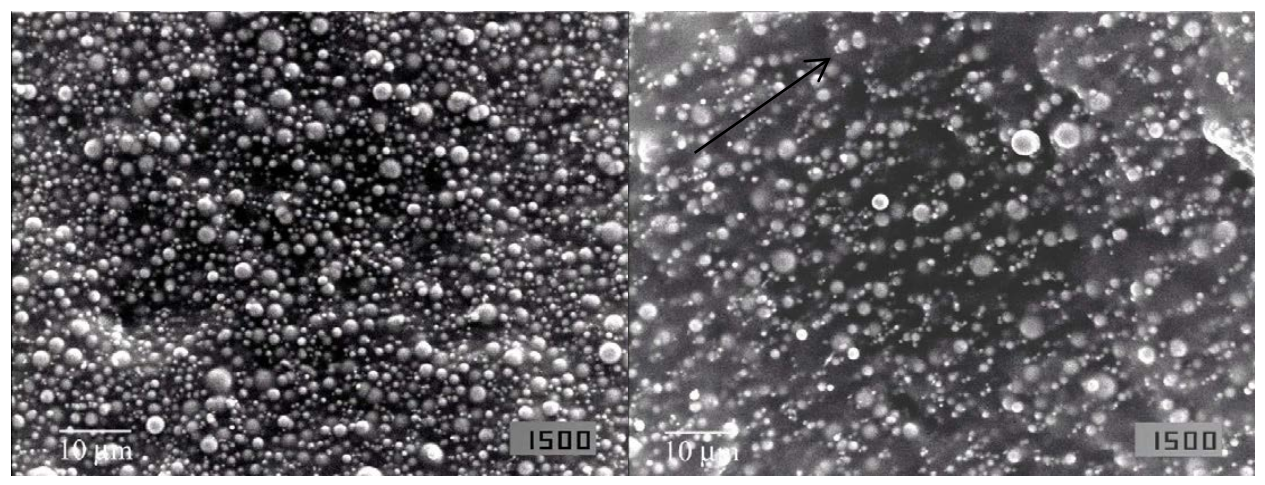

(a)

(b)

Figure 2. Scanning electron micrographs of a fracture surface of (a) an isotropic MRE with 30 vol\% of Fe and (b) an anisotropic MRE with $30 \mathrm{vol} \%$ of Fe, magnification $1500 \times$. The arrow points to the alignment direction. [22] 
The most distinctive change in material property of MR elastomer under magnetic field is its modulus [9]. Yet in some studies it is stated that damping property of the material also changes due to magnetic field, other studies concluded that magnetic field does not have any remarkable effect on damping property [14, 20, 23- 28]. Majority of the damping may come from the frictional sliding at the interfaces between the free rubber and the particles [29]. As such, the property of MR elastomer is normally evaluated by the MR effect induced, which is the ratio of modulus increase $\Delta \mathrm{G}$ at measured magnetic field to the initial storage modulus G0 at zero magnetic field.

In the pilot work by Ginder et al [30], it is suggested that MR elastomer can be used to develop tuned vibration absorbers (TVAs). Since then, research on MR elastomer has progressed to a new level where significant amount activities have been reported, including material empirical modeling [18, 31-40], material development and property testing [2-3, 1012,14-15, 18, 21,41-60], new device design and characterization [61-84], and performance evaluation [85-105] and aimed for applications in aeronautic engineering, mechanical engineering, automobile engineering and civil engineering. In general, MR elastomer has attracted broad attention with its unique property and its potential in active or semi-active vibration control, such as for vibration or/and noise reduction and vibration isolation, attributing to its inherent material properties, i.e. large modulus change [68], fast response time (faster than MR fluids) [1-3], stability, compatibility to mechanical components, reasonable low power requirement. In particularly, wide range of adjustable modulus offers more effective means than semi-active damping associate with MR fluids devices to mitigate vibration disturbances [20]. Creative designs of devices incorporating MR elastomer have been reported for vibration absorbers [62-71], vibration isolators [16,72,88-89], adaptive base isolators [20,78-79,93], vibration mount [76] and sandwich beam [82-84, 95-103], aimed for various applications, i.e. vehicle seat vibration suspension, adaptive base isolation, and so on.

There have been efforts in reviewing the technological developments in MR elastomer technology [17, 106], which mainly focused on material development rather than MR elastomer device development due to the early stage of related developments when published. To accommodate the fast growing demand and to identify the challenges in this research area and to identify the challenges in the field, this paper attempts to provide a timely state-of-theart review on the MR elastomer technology with special focus on material preparation and property, device design and analysis, experimental testing techniques, and nonlinear performance modeling of the devices. The paper will review basic design and related issues of the MR elastomer devices, summarize and comment on developments and applications of creative design of MR elastomer devices to date and then discuss on challenges and opportunities to be addressed for the future research in the area.

\section{Basic design issues of MR elastomer devices}

\subsection{Operation modes}

Understanding operation modes of MR elastomer material is essential step towards design of devices. Operation modes of MR fluids have been discussed extensively and understood well [1, 107-108]. They refer to combinations of deformations of material and chain structure directions. For MR fluids, iron particles can move freely in the carrier oil and hence the 
direction of the chain structures aligns with direction of magnetic field. However, for MR elastomers, iron particles are locked within the polymeric matrix and external excitations, i.e. deformation and magnetic field, and only leads to local movement around original locations. Therefore, the direction of the chain structures no longer coincides with magnetic field. Besides, as soft elastic material with magneto-active property, MR elastomer deforms along magnetic field [75]. Above properties make operation modes of MR elastomers quite different from these of MR fluids.

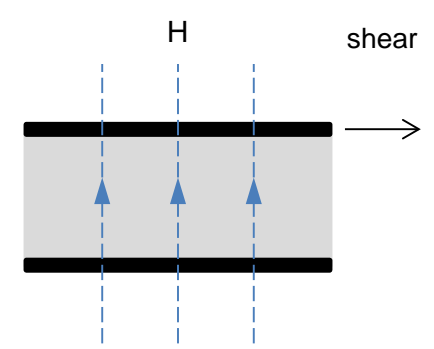

(a)

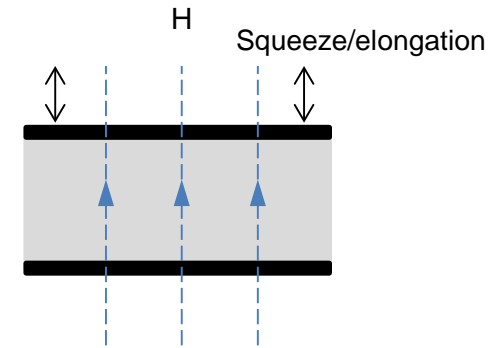

(b)

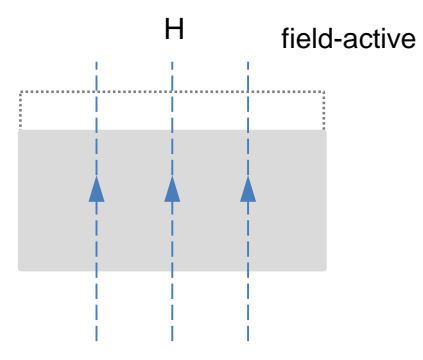

(c)

Figure 3. Basic operation modes for MR elastomers: (a) shear mode; (b) squeeze/elongation mode; and (c) field-active mode;

Figure 3 illustrates three basic operation modes of MR elastomers: shear mode, squeeze/elongation mode and field-active mode. Note that as solid material, pressure mode (or flow mode) which works for MR fluids is not applicable for MR elastomer. However, in field-active mode, MR elastomers can change their shapes, i.e. being stretched by magnetic field. This property is called as magnetostriction [27,109]. For shear mode and squeeze/elongation mode MR elastomers work similarly as MR fluids. Both isotropic and anisotropic MR elastomers can be used for the three basic operation modes. Therefore, different configurations can be found for devices with isotropic and anisotropic MR elastomers, i.e. combinations of field directions and aligned chain directions. MR elastomer working in field-active mode can be used to design various actuators [75, 77]. Examples of shear mode devices include vibration absorbers [62-70], vibration isolators [16, 71, 89-90] and base isolators [20, 78-79, 93]. Examples of squeeze/elongation mode devices are vibration absorbers [64-65], engineering mounts [76] and compressive spring elements [22].

\subsection{Magnetic circuit design}

To provide a controllable magnetic field, electromagnetic coil or solenoid, is normally used for MR fluid devices and MR elastomer devices. Altering the applied current can change the magnetic induction through the field-sensitive materials. An effective magnetic circuit design provides MR elastomer devices the optimal MR effect. Ideally, the magnetic field flux should be perpendicular to the motion of the MR fluids and MR elastomers. In such way, the MR effect can be fully utilized.

The most effective and efficient magnetic circuit design is the C-shape magnetic circuit since it creates an enclosed field path for the magnetic flux and thus has minimum energy losses. Example of C-shape magnetic circuits can be found in [16-17, 64]. To eliminate the energy losses in the air as well as maintain uniform and strong magnetic field, the gap between two poles of the C-shape magnetic circuit maintains as small as possible. Such requirement leads 
to small size of material to be effectively energized. In addition, a significant length and scale of other part of the C-shape design, such as coil and conductive path, is inevitably required. Therefore, efficient and compact magnetic circuit design is a challenge for designing MR elastomer devices.

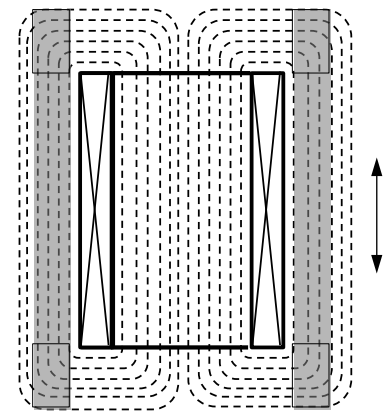

(a)

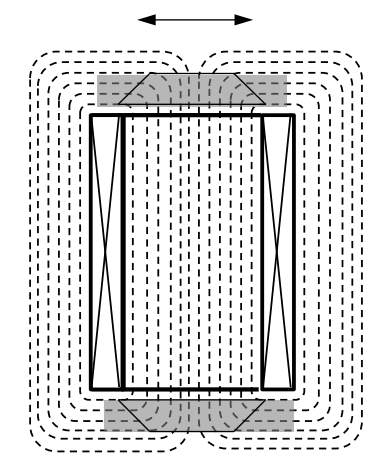

(b)

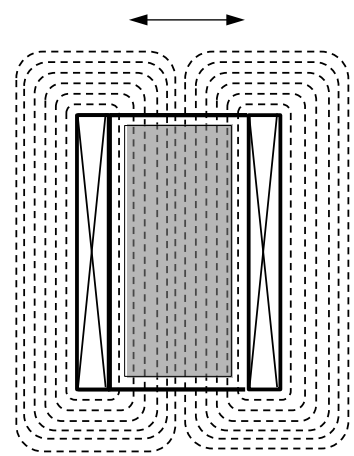

(c)

MR materials $\square$ Magnetic flux line $\square$ Effective area $\longrightarrow$ Motion direction

Figure 4. Three configurations for magnetic circuit design: The shadow areas indicate the field-sensitive materials. The active areas are embraced within solid line. Magnetic flux is indicated with dash line. The motion directions of the smart devices are also labeled with arrows.

Generally, there are three configurations for a magnetic circuit design for the devices towards application, as shown in figure 4. In the first configuration, the field-sensitive material is placed outside the magnetic coil and the motion direction of the material is parallel to the axial of the coil. Most of the MR fluid damper designs used this configuration [110]. However, due to the requirement on the field direction, only small sections of MR fluids in active area exhibit MR effect. The active area is normally very narrow in the way of flux line due to the low magnetic permeability of MR fluids and large force requirement of the device design [110]. Given the gap between the cylinder and piston surface is very small (around $0.5 \mathrm{~mm}$ to $1.0 \mathrm{~mm}$ ), provision of a saturated field for the MR fluid in between is not difficult to achieve. However, comparing to small amount MR fluids in the active areas, majority of the MR fluids in the flow channel are energized in the direction paralleling to the flow direction. Although this does not impose a problem for MR fluid devices such as MR damper since blockage of the flow in a narrow channel creates large damping effect, for MR elastomer devices, it is can be a problem since the devices often require to energize a large portion of MR elastomers.

In the second type of magnetic circuit configuration as shown in figure 4, the MR materials are closely configured on the top or/and the bottom of the solenoid. To make the best use of the magnetic field, the materials are normally attached on the surface of the coil. Many designs of MR elastomer-based vibration absorbers and vibration isolators adopted this configuration. Examples of such design can be found in [72, 94, 111]. Behrooz et al [93, 111] proposed a MR elastomer vibration isolator with optimized shape under this configuration, as shown in figure 24. After the finite element simulation, four trapezoid MR elastomer samples were used between four magnetic coils to best use the magnetic field flux path. Similar as the 
first configuration, the active area is small and energizing a thin layer of MR material will require considerable additions, such as space for the magnetic coil and magnetic path.

In the third type of the configuration the MR materials can be placed inside of the magnetic coil, serving as the magnetic core of the magnetic circuit. Comparing with the weak and divergent magnetic field outside of the coil, the magnetic field inside the solenoid is strong and uniform [20, 78, 112-113]. The greatest advantage of this design is that it provides a large active area and strong magnetic field. The MR materials inside the solenoid can be fully energized by a uniform magnetic field. However, due to the low permeability of the MR elastomer material, special measures should be taken to increase the permeability of the magnetic core for which MR materials form a part of it. Kallio et al [22] adopted this configuration in a squeeze mode tunable spring element using MR elastomer. In his design, the magnetic coil device was designed so that the spring element, MR elastomer material, is placed inside the coil formed part of the closed magnetic circuit, as shown in figure 12 . One steel cylinder was placed under the MR elastomer element to increase the magnetic conductivity of the magnetic core. The MR elastomer was applied with compressive loading in the direction aligned with the magnetic flux line. Li et al [20, 78-79] designed MR elastomer base isolators in which the laminated structure containing multiple MR elastomer layers and steel blocks serve as the core of the magnetic circuit, figure 26. In the works by $\mathrm{Li}$ et al 2013 [78-79], the magnetic field achieved inside the 94mm thick MR elastomer with diameter of $140 \mathrm{~mm}$ is up to $0.3 \mathrm{~T}$. A magnetic field well above $0.44 \mathrm{~T}$ has also generated for 25 layers of MR elastomer with diameter of $120 \mathrm{~mm}$ and overall thickness of $25 \mathrm{~mm}$ in their later work [20].

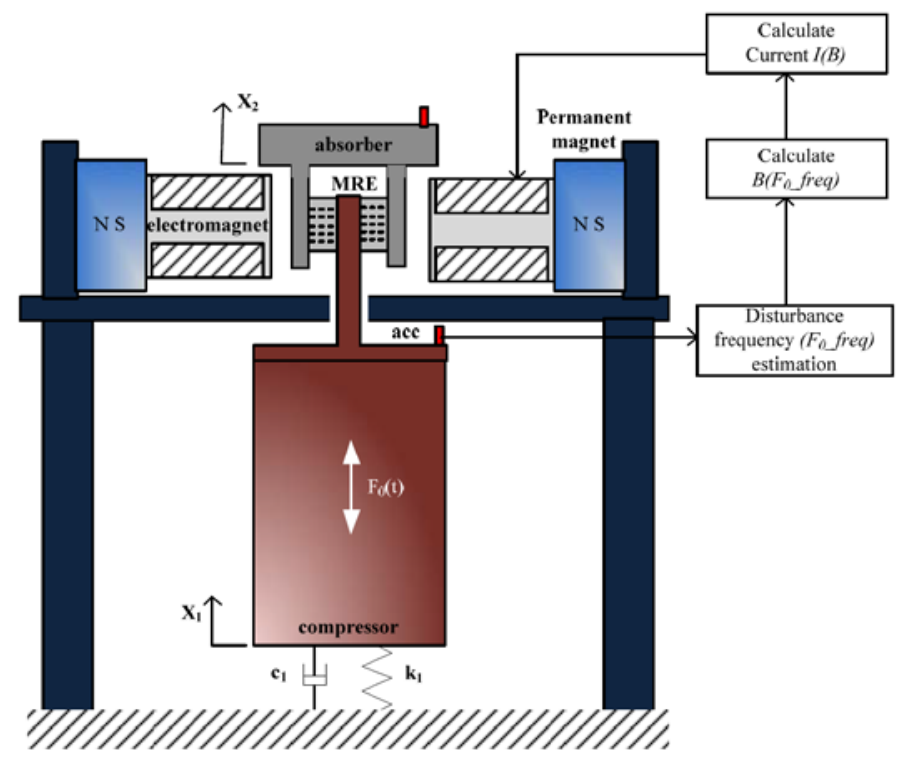

Figure 5 Adaptive MR elastomer based tunable vibration absorber using hybrid magnetic design [69]

Most of the variable stiffness MR elastomer devices use magnetic field to enhance the stiffness of the device. Hybrid magnetic design has been used to realize negative stiffness in the MR elastomer devices. Kim et al [69] designed a tunable vibration absorber using both permanent magnetic and electromagnetic coil for field generation, figure 5. Sinko et al [70], Opie and Kim [72] also reported their designs of vibration absorber and vibration isolator 
with same idea. By applying positive and negative current, the magnetic field can be enhanced or weakened towards the existing magnetic field generated by permanent magnetics. Such design is particularly valuable when certain stiffness is required for the device to maintain serviceability and stability of structures.

\subsection{Fundamentals of design}

MR elastomer vibration absorbers and isolators may work in shear mode, squeeze/elongation mode or combined mode of those two. As variable stiffness devices, their effects are to alter natural frequencies as:

$$
\omega_{n}=\sqrt{\frac{K}{M}}
$$

Where, $K$ and $M$ are the stiffness and the lumped mass of the device, respectively.

Adding damping in the system, such as by using MR fluid damper, is less efficient to change natural frequency of the device since:

$$
\omega_{d}=\omega_{n} \sqrt{1-\zeta^{2}}
$$

Where, $\omega_{d}$ is the damped natural frequency after adding damping into the system and $\zeta$ is the damping ratio of the system. For example, for a high damping ratio at $\zeta=20 \%$, the damped natural frequency is $0.979 \omega_{n}$, which only about $2 \%$ difference from the undamped natural frequency.

Performances of the devices can be achieved by evaluating its fundamental frequency which relates to the stiffness (refers to static stiffness here and later in this paper) of the devices. For shear mode device with cross-section area of MR elastomer layer as shown in figure 6, the stiffness can be expressed as:

$$
K_{s}=\frac{G A}{n h}
$$

Where $G$ is the shear modulus of MR elastomer, $\boldsymbol{A}$ and $\boldsymbol{h}$ are the cross-section area and the thickness of the rubber layer, respectively; $n$ is the number of MR elastomer layers in the device.

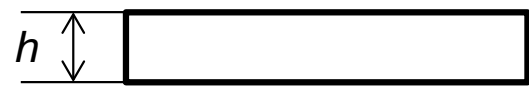

Figure 6. Cross-section view of the rubber layer

For squeeze mode MR elastomer devices, the compressive stiffness of the device can be expressed as:

$$
K_{s}=\frac{E A}{n h}
$$

Where $\boldsymbol{E}$ is the compression modulus of the material. 
For single degree-of-freedom (SDOF) base vibration isolation system shown in figure 16 (a), the vibration transmitted to isolation mass can be expressed by the displacement transmissibility and force transmissibility as:

$$
\begin{gathered}
\frac{X}{Y}=\sqrt{\frac{1+(2 \varsigma r)^{2}}{\left(1-r^{2}\right)^{2}+(2 \varsigma r)^{2}}} \\
\frac{F_{T}}{k Y}=r^{2} \sqrt{\frac{1+(2 \varsigma r)^{2}}{\left(1-r^{2}\right)^{2}+(2 \varsigma r)^{2}}}
\end{gathered}
$$

Where $r=\frac{c}{2 m \omega_{n}}$ and frequency ratio $\varsigma=\frac{\omega}{\omega_{n}}$

While for SDOF force isolation system shown in figure 16 (b), the force transmissibility is expressed as:

$$
\frac{F_{T}}{F_{0}}=\sqrt{\frac{1+(2 \varsigma r)^{2}}{\left(1-r^{2}\right)^{2}+(2 \varsigma r)^{2}}}
$$

\section{Magnetorheological elastomer devices}

Differences between MR elastomer devices and MR fluid devices can be summarized as followings: (1) MR elastomer devices work in pre-yield region while MR fluid devices work in post-yield region. Thus MR elastomer devices mainly have variable stiffness while MR fluid devices mainly possess variable damping; (2) Operation modes are also different for them, see section 3.1 for details. (3) MR elastomer device can work in multiple degree-offreedoms (DOF) while MR fluid devices merely work in single DOF [72, 85-88]. Possessing variable stiffness and damping subjected to magnetic field, MR elastomer is natural candidate to be developed into smart devices, such as vibration absorbers, vibration isolators, base isolators as well as sandwich beams.

\subsection{Vibration absorbers}

Vibration absorbers, also called as tuned vibration absorbers (TVAs) or dynamic vibration absorbers (DVAs), are to attenuate the vibrations of the primary structure (figure 7) which is often excited by rotational imbalances from engines, motors, and pumps [114-115]. Passive TVAs only work effectively for limited range of frequencies as designed. When excitations are outside of the designed frequency it causes the loss of effectiveness or even amplifies the vibration of the system. For such reason, it is desirable that the device can adjust its designed frequency accordingly [115]. MR elastomers allow the development of adaptive vibration absorbers (AVAs) as a result of its field-dependent stiffness property which can be used to vibration absorption for a wider range of frequencies. Frequency shifting capability is the main measure for TVAs and adaptive tuned vibration absorbers (ATVAs) and transmissibility suits for SDOF systems.

Ginder et al [30] prototyped the first MR elastomer TVA working in shear mode and experimentally tested its performance, figure 8. The MR elastomer TVA was constructed using a closed magnetic circuit which was formed by metallic part made of low-carbon steel, a wired coil for field generation and two anisotropic MR elastomer samples. The reaction mass was mounted between two samples to allow vertical motion. A wired coil was placed 
around one arm which naturally concluded that adding one more aired coil around another arm would have further strengthened the magnetic field intensity inside the MR elastomer samples. MR elastomer samples were fabricated at a volume fraction of $27 \%$ under a magnetic field of $0.8 \mathrm{~T}$. Size of the sample was rectangular with size of less than $60 \mathrm{~mm}$. Experimental testing on transmissibility showed that the resonant frequencies of the system can be shifted from around $500 \mathrm{~Hz}$ to above $600 \mathrm{~Hz}$ when the magnetic flux densities varied from 0T to 0.56T. Deng et al [19] improved the design by Ginder et al [30] by adding another wired coil along the magnetic path, figure 9. For this design, the directions of two coils should be arranged in such way to strengthen the magnetic field. Anisotropic MR elastomers with iron particle of $70 \%$ mass ratio were used in the design. Resonant frequency of the device can be controlled by electrical currents from $55 \mathrm{~Hz}$ at $0 \mathrm{~A}$ to $81.25 \mathrm{~Hz}$ at $1.5 \mathrm{~A}$, equivalent to an increase of $147 \%$. Effectiveness of the ATVA was evaluated by a two-endsupported beam structure. Utilizing similar configuration, Zhang and Li [66] developed an ATVA using two anisotropic MR elastomer samples of 3mm thick and the proposed vibration absorber is able to change its natural frequency from $35 \mathrm{~Hz}$ at applied current of $0 \mathrm{~A}$ to $90 \mathrm{~Hz}$ at current of 3A. Numerical evaluation on its vibration reduce efficiency was undertaken based on the device performance. Results showed that the proposed ATVA can trace the disturbance force frequency and absorber the inertia energy transmitted from the primary structure and thus suppress the vibration. Other shear mode ATVAs can be found in the works done by Liao et al [16], Kim et al [70] and Xu et al [67]. A compact shear mode AVTA using MR elastomer was designed and tested by Deng and Gong [62-63], shown in figure 10. The electromagnets and magnetic conductor form closed C-shape magnetic path while also serve as dynamic mass. In this way, the device has more compact configuration than the previous ones. Experimental testing proved that MR elastomer can tune the natural frequency of the device from $27.5 \mathrm{~Hz}$ to $40 \mathrm{~Hz}$.

The shear mode ATVAs can also be used to suppress torsional vibration. Hoang et al [85-87] proposed a MR elastomer TVA for powertrain vibration suppression, figure 11 . The rotational part of the device consists of an outer ring, inner ring and $8 \mathrm{MR}$ elastomer specimens located in the gap between the rings. Two mild steel covers are used to lead the magnetic flux to the MR elastomer materials. With such arrangement, the magnetic field passes through the MR elastomer in the direction as indicated in figure 11. However, it should be noted that the shear motion is not perpendicular to the magnetic field, which may reduce the MR effect and thus downgrade the performance of the device.

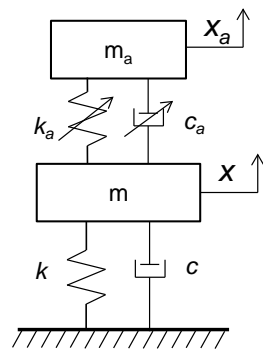

(a)

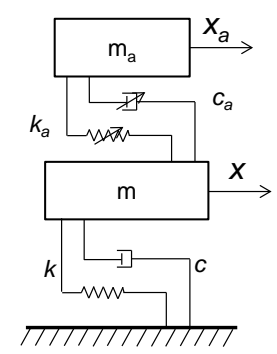

(b)

Figure 7. Sketch of dynamic vibration absorption systems featuring MR elastomers: (a) for vertical vibrations; (b) for lateral vibrations. Here, $\mathrm{m}$ is mass of primary structure and $\mathrm{m}_{\mathrm{a}}$ is the mass of absorber. 


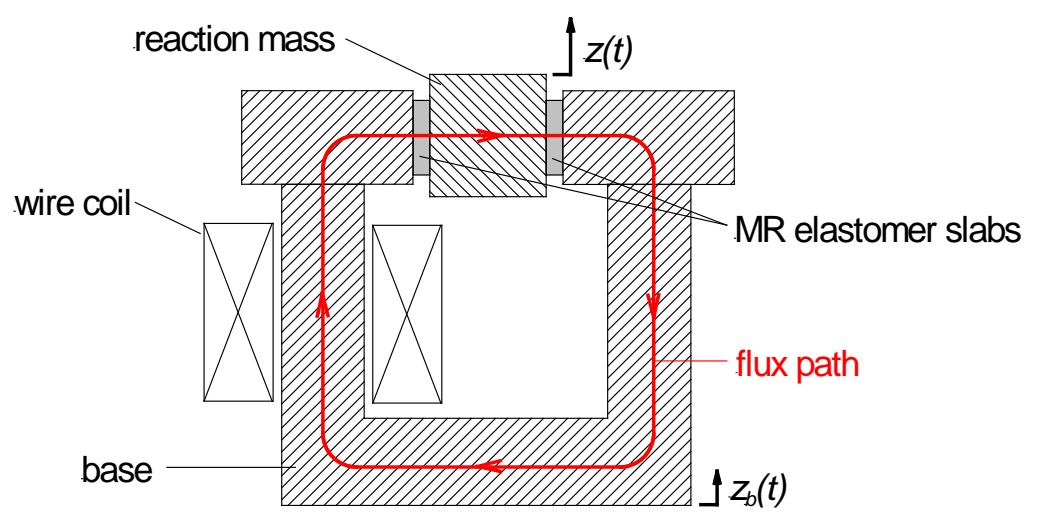

Figure 8. MR elastomer TVA designed by Ginder et al [30] from Ford Research Laboratory

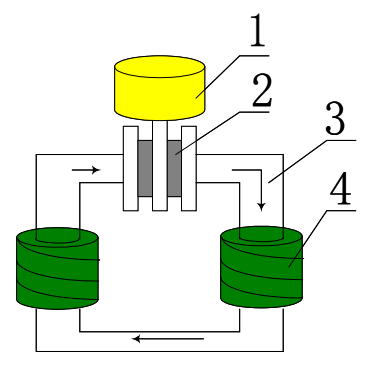

(a)

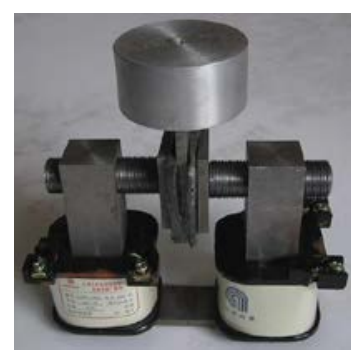

(b)

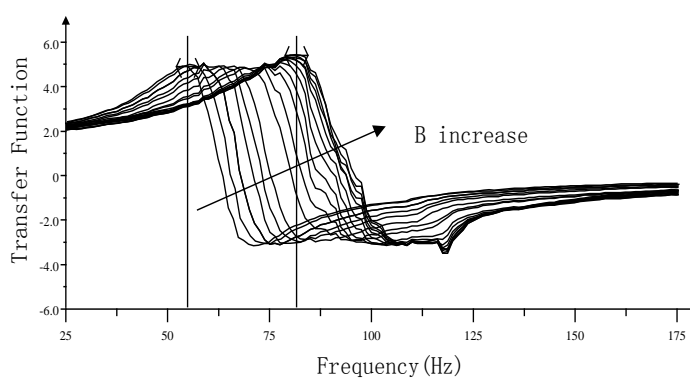

(c)

Figure 9. Adaptive tuned vibration absorber proposed by Deng et al [19] and its performance: (a) Sketch: 1. Oscillator; 2. MR elastomers; 3. Magnetic conductor; 4. Coils; (b) Photograph; (c) The transfer function versus frequency at various magnetic fields. (Acknowledgement: Original figures provided by corresponding author, Prof. X L Gong, from University of Science and Technology of China)

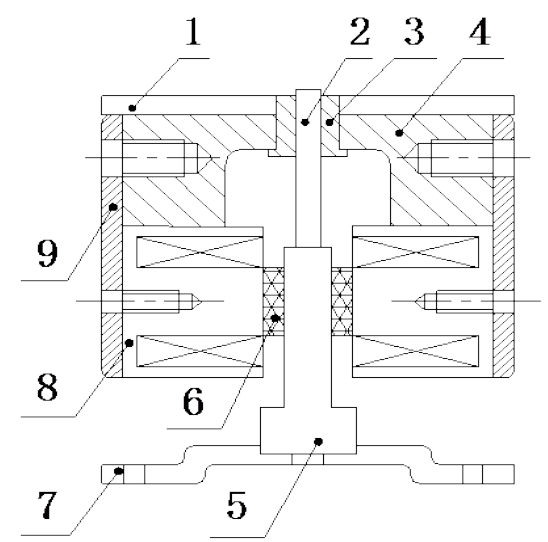

Figure 10. A compact MR elastomer ATVA designed by Deng and Gong [62]:1. cover; 2. guide rod; 3. linear bearing; 4. magnetic conductor; 5. shear plate;6.MREs; 7. base; 8. electromagnet; 9. mounting shell. (Acknowledgement: Original figure provided by corresponding author, Prof. X L Gong, from University of Science and Technology of China) 

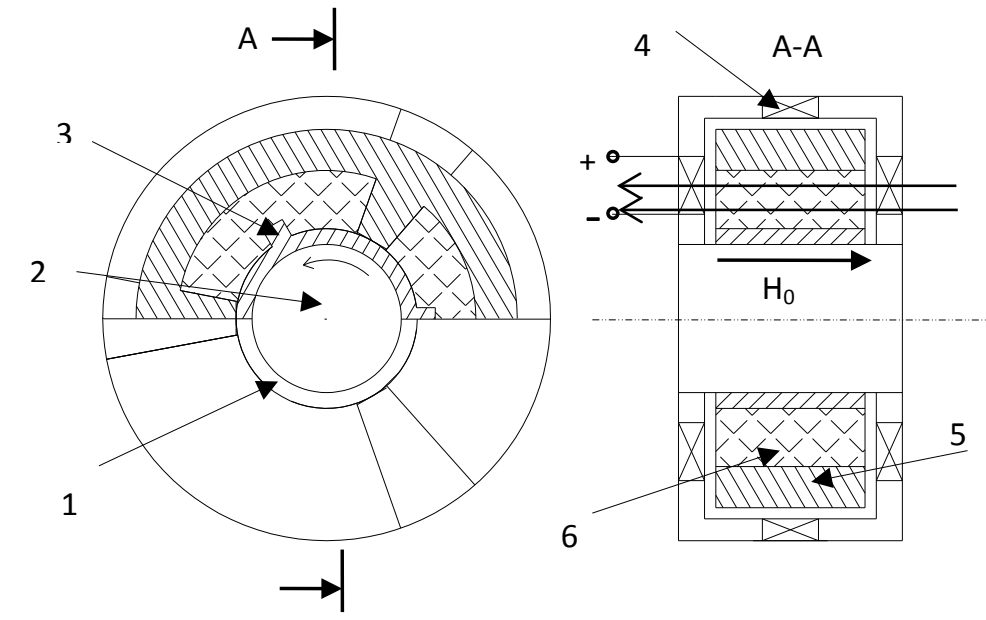

Figure 11. Torsional vibration absorber designed by Hoang et al [85-87]:1.inner cylinder; 2. rotating shaft; 3. lug; 4. electromagnetic coils; 5 .outer cylinder; 6 . MR elastomer material.

Kallio et al [22] proposed a tunable spring element utilizing MR elastomer, shown in figure 12. The device works in squeeze mode and allows compressive loading applied on it. Magnetic field adjusts its compressive modulus thus presents it tunable property. Lerner and Cunefare [64] proposed three kinds of MR elastomer based vibration absorbers (figure 13) to be used in ATR 42 and 74 aircraft fuselages, i.e. shear device, longitudinal device and squeeze model device. As can be seen, shear device operates in shear mode while other two devices work in squeeze/elongation mode. C-shape magnetic circuit design is adopted for all three vibration absorbers. Despite arranged in different modes, basic components in the vibration absorbers are base mass, absorber mass, MR elastomer and wire coil. Figure 13 illustrated the designs of the vibration absorbers and the arrows in the figure indicate the direction of the excitations. Anisotropic MR elastomer samples in the work were composed of $35 \% \mathrm{vol}$ iron particles. Experimental testing demonstrated that the shear device and longitudinal device have natural frequency increases of $183 \%$ and $473 \%$, respectively, between field intensity of 0 and $183 . \mathrm{kA} / \mathrm{m}$. While the squeeze mode device have maximum natural frequency increase of 507\%. Squeeze mode MR elastomer absorber can also be designed in a more compact manner, shown in figure 14 designed by Sun et al [71]. MR elastomer material with mass fraction ratio of 7.5: 1.25: 1.25 (iron: silicon oil: silicon rubber) was used in the device. Magnetic coils and steel components form dynamic mass and were placed on top of the MR elastomers. The results revealed that the squeeze MR elastomer absorber extended its vibration attenuation range from $37 \mathrm{~Hz}$ to $67 \mathrm{~Hz}$.
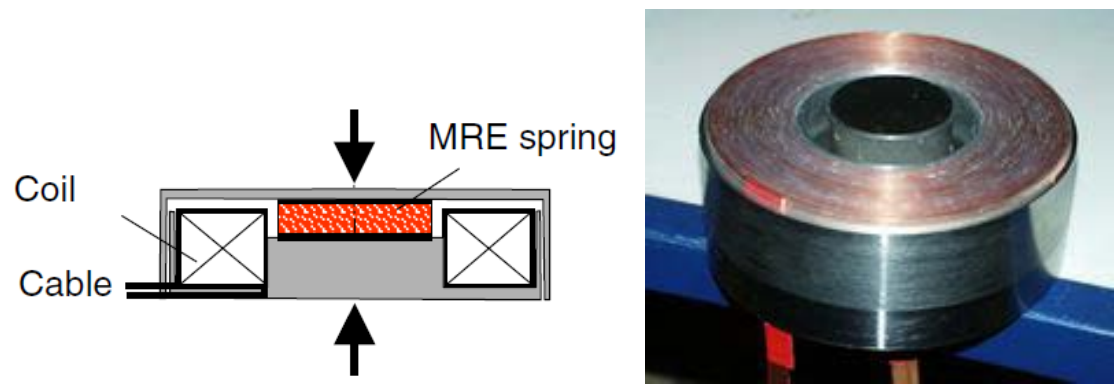

Figure 12. Tunable spring element proposed by Kallio et al [22] 

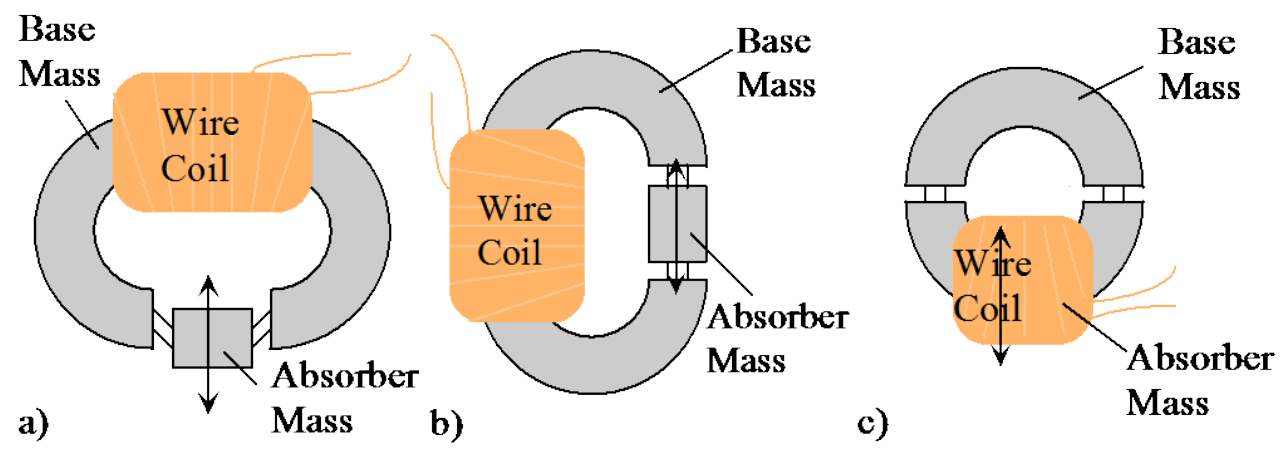

Figure 13. Three vibration absorbers designed by Lerner and Cunefare [64]: (a) shear device; (b) longitudinal device; and (c) squeeze mode device with direction of excitation (indicated by arrows).

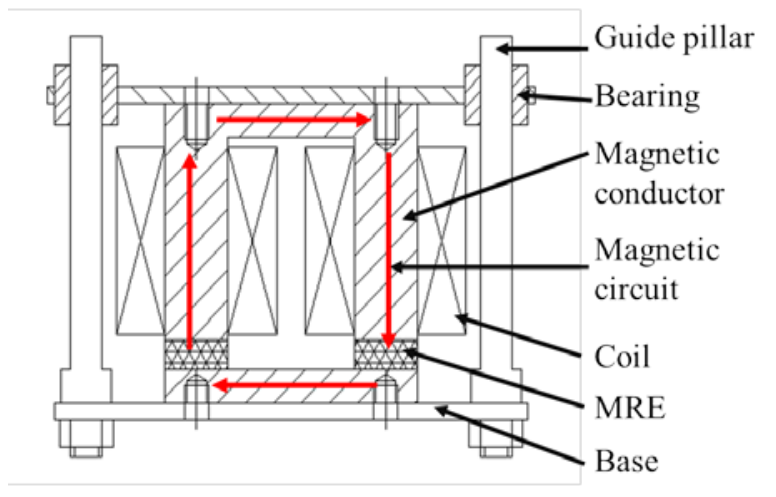

Figure 14. Schematic of a compact squeeze MR elastomer absorber designed by Sun et al [71] (red line indicate the magnetic flux path).

ATVAs can also be designed with combined mode, such as combining shear mode and squeeze mode. It is revealed that the MR effect in shear under compressive loading is larger than that in pure shear mode [3,64]. Utilizing such understanding, Ni et al [65] designed an MR elastomer AVTA in combined shear and squeeze modes, figure 15. In this design, piezoelectric actuator installed on a movable arm was to apply controllable compressive loading to the MR elastomer samples. Numerical simulations indicate that such design realizes more effective suppression capabilities than that in shear mode only.

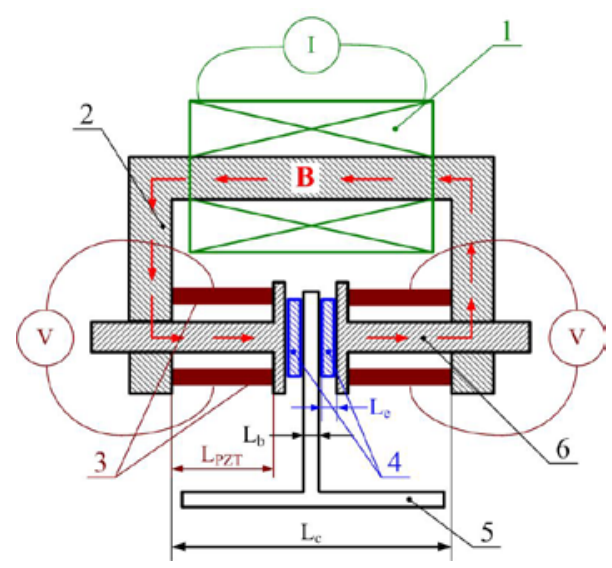

Figure 15. Dynamic stiffness-tuning absorber with squeeze-strain enhanced MR elastomer, designed by Ni et al [65]:1. Excitation winding; 2. C-shape magnetic conductor; 3. Terrace PZT actuator; 4. MRE; 5. Base; 6. Moveable arm. (Acknowledgement: Original figure provided by corresponding author, Prof. X L Gong, from University of Science and Technology of China) 


\subsection{Vibration isolators}

Vibration isolation is to isolate the vibration sources from one to the parts by install vibration isolator between them, figure 16. It is categorized into two groups: base isolation and force isolation [116]. Although majority of the MR elastomer vibration isolators discussed in this paper are used for base isolation, they could be equally used for force isolation as well. Also note that despite that figure 16 only shows a MR elastomer isolator of vertical direction, vibration isolators can work in both lateral and vertical directions in terms of isolating vibrations. Displacement transmissibility and force transmissibility are the references to evaluate the system performance of SDOF system (detailed discussion can be found on section 2.3).

\subsubsection{Vibration isolators for mechanical engineering application}

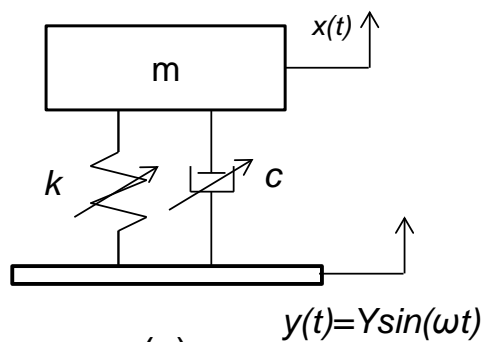

(a)

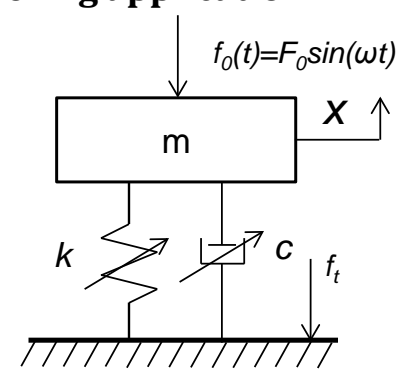

(b)

Figure 16. Principle of vibration isolation systems: (a) base motion isolation; (b) force isolation;

Passive isolators work well for designed narrow bands of frequencies, the performance of the isolators deteriorate when source excitations are outside such designed frequency bands. Main reason for such limitation is that a designed isolation frequency for passive isolators is a constant which is fixed mass and stiffness of the device. On the other hand, a MR elastomer vibration isolator, possessing controllable stiffness, enables it to adjust its isolation frequency in real time. Suspension bushing, engineering mount and seat vibration isolator are examples of such applications in the area.

Ginder et al [27] used MR elastomers to design tunable automotive bushing for vibration reduction on translational and rotational motion of wheels, figure 17. The bushing consists of two concentric hollow cylinders made of steel. Between the cylinders is the annular MR elastomer fills with height of $60 \mathrm{~mm}$, inner radius of $17 \mathrm{~mm}$ and outer radius of $28 \mathrm{~mm}$. An electromagnetic coil of 300 turns is mounted on the inner hollow cylinder to generate magnetic field for energizing MR elastomers. Sinusoidal deformation testing was conducted at $2 \mathrm{~Hz}$ and $2 \mathrm{~mm}$ peak-to-peak amplitude. Both stiffness and damping in axial and radial directions exhibited a 25\% linear increase with applied current up to 5A. Liao et al [16] proposed a tunable stiffness and damping vibration isolator using MR elastomer, figure 18. This design is configured similarly with works in [30] and [19]. It consists of eight parts: base, magnetic coils, magnetic conductor, shear plate, iron core MR elastomer, voice coil motor and mounting plate. Three coils were used to generate magnetic field for 4 layers of MR elastomers to provide tunable stiffness. The voice motor was used to generate force which is proportional to the shear velocity. Under such mechanism, tunable stiffness and damping are 
achieved. Kavlicoglu et al [76] proposed a MR elastomer mount, shown in figure 19. The MR elastomer mount is developed by using 0.5-inch thick MR elastomer layers and two built-in electromagnets. Two mounting plates are placed on top and bottom serving both for mechanical connection as well as magnetic path. The performance of the 2-layer MR elastomer mount is characterized by compression, shear, vibration, and shock tests. However, magnetic path in this design is not a closed loop and thus the magnetic design is not efficient.
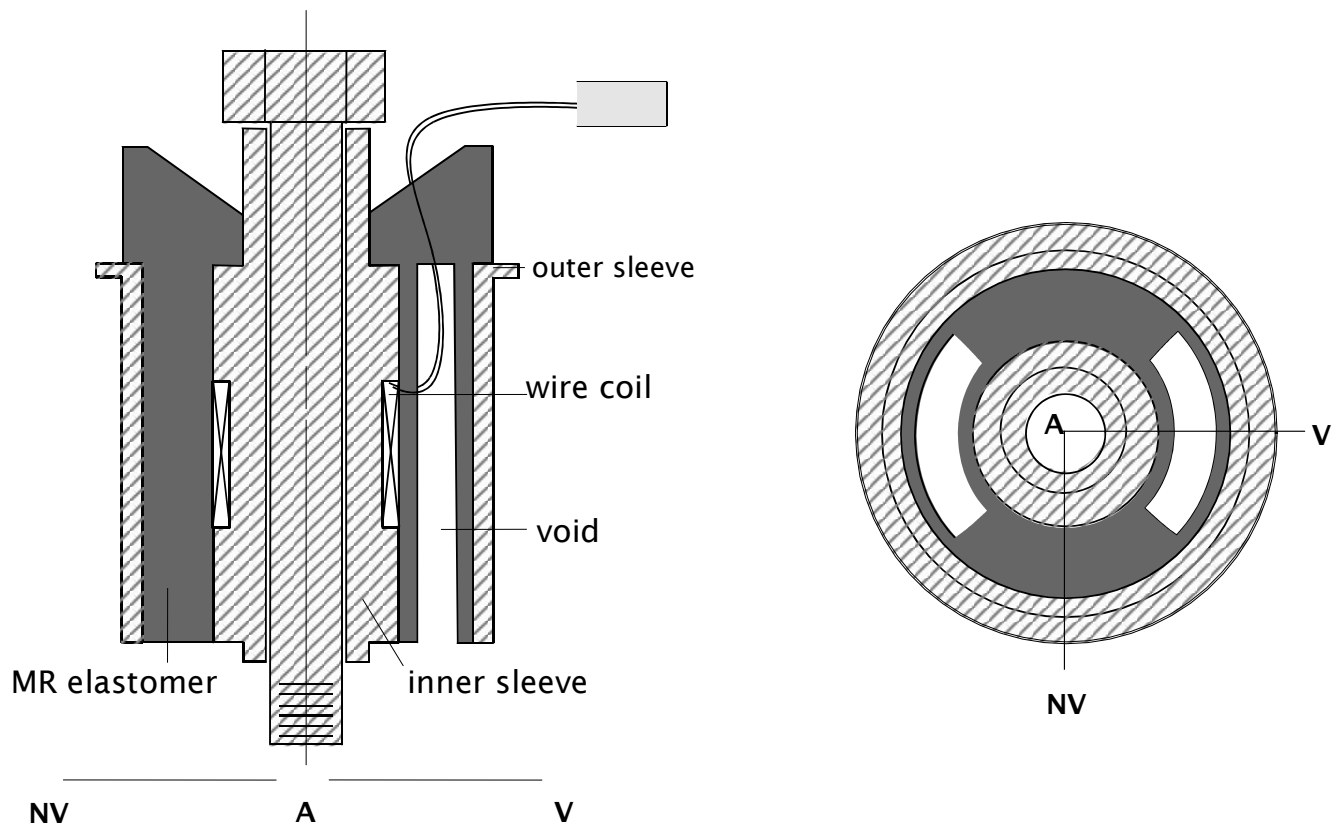

Figure 17. Schematic section view of MR elastomer bushing by Ginder et al [27]: The sectional view is along the cut NV$\mathrm{AV}$ where NV denotes the non-voided direction, $\mathrm{V}$ denotes the voided direction, and A denotes the axis of rotation of the bushing.

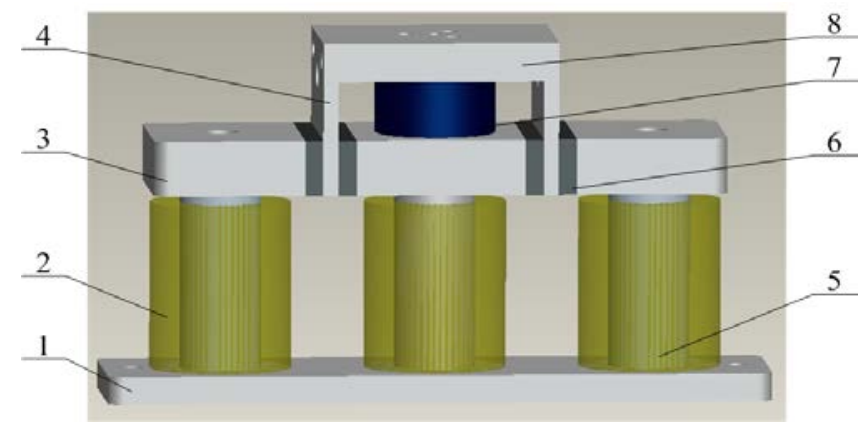

Figure 18. Schematic of MR elastomer based vibration isolator designed by Liao et al [16]:1, base; 2, magnetic excitation coil; 3, magnetic conductor; 4 shear plate; 5, iron core; 6, MRE; 7, voice coil motor; 8, mounting plate. (Acknowledgement: Original figure provided by corresponding author, Prof. X L Gong from University of Science and Technology of China) 


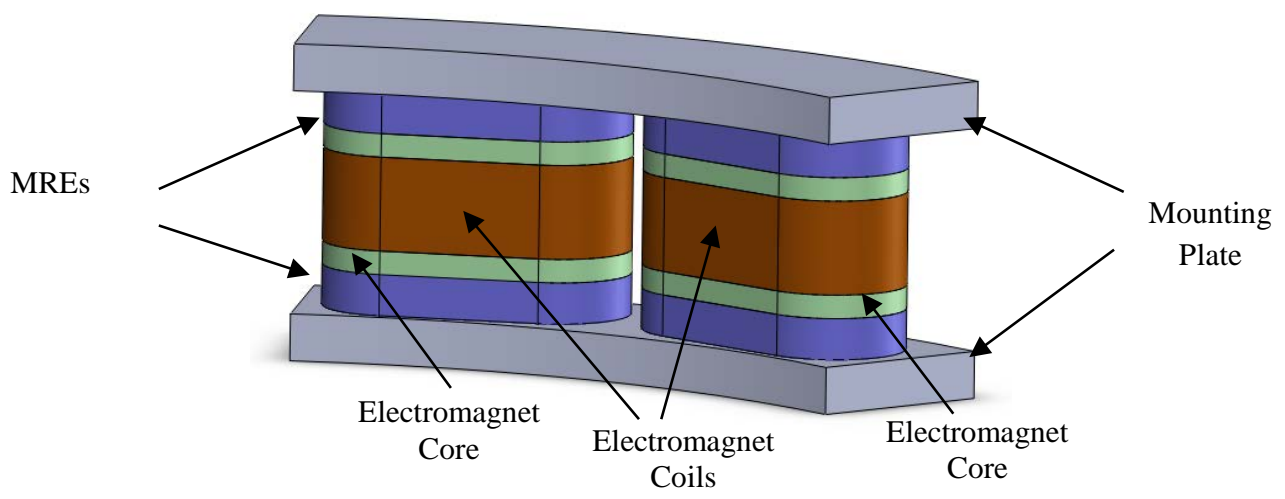

Figure 19. MR elastomer mount proposed by Kavlicoglu et al [76]

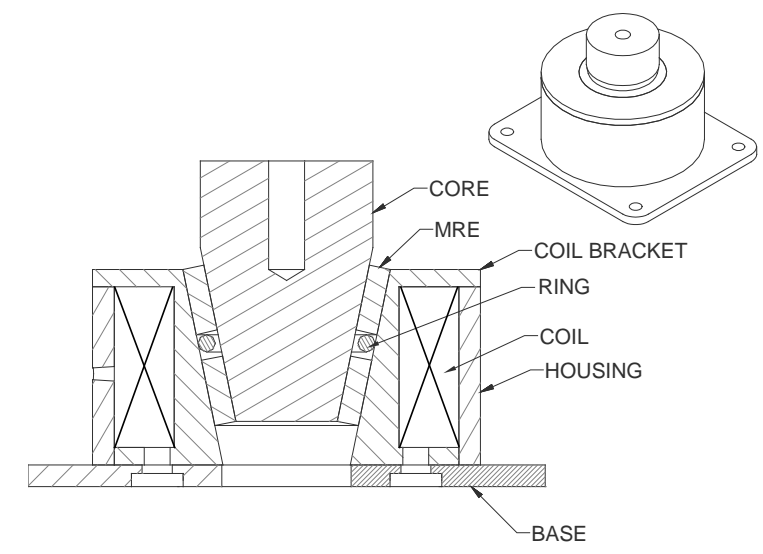

Figure 20. Schematic of the magnetorheological elastomer seat isolator by Li et al [91]

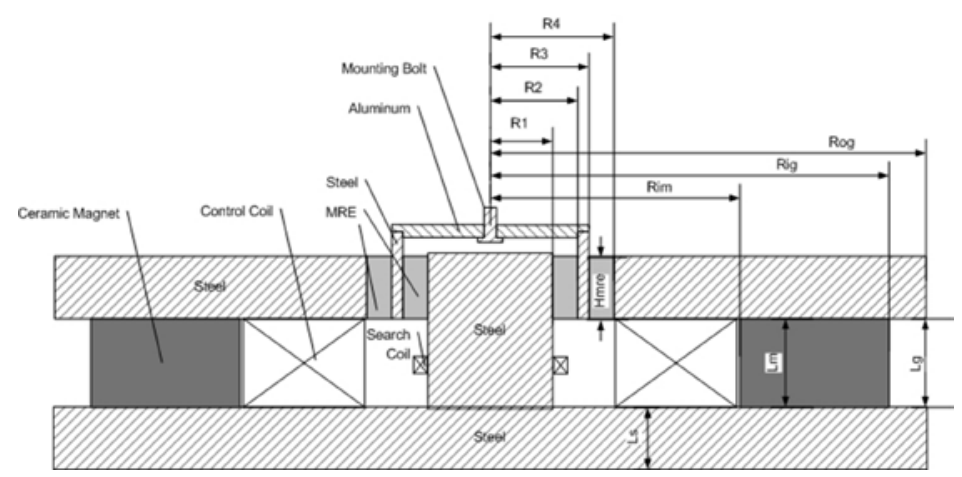

Figure 21. MR elastomer vibration isolator designed by Opie and Yim [72] 


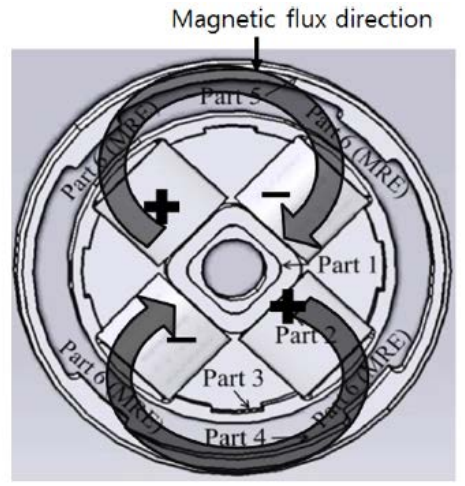

(a)

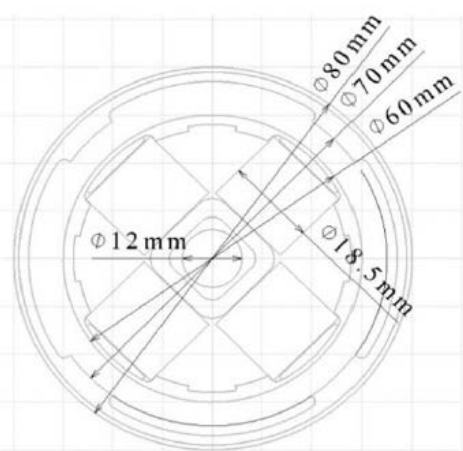

(b)

Figure 22. Stiffness-variable differential mount proposed by Jeong et al [117]: (a) schematic geometry and (b) engineering drawing

$\mathrm{Li}$ et al [90] and Du et al [91] developed a new MR elastomer isolator to be used for seat vibration control in vehicles, figure 20. This device is composed of core and base, magnetic coil, non-magnetic rings and MR elastomer. The coil, core and base form the magnetic path. The proposed seat isolator works in both shear and compressive mode. Experimental testing showed this device exhibited clearly increase on stiffness and damping. Opie and Yim [72] designed a MR elastomer vibration isolator using hybrid magnetic circuit, figure 21. This device mainly consists of steel frame, steel core, ceramic magnetic and electromagnetic coil. The implementation of the permanent magnetic allows the magnetic field inside MR elastomer to be increased or decreased by applying negative and positive currents in the coil. Jeong et al [117] designed a MR elastomer differential mount with variable stiffness to be used in vibration isolation between engine and car body of vehicles, figure 22. Considering the hostile environment in the vehicle system, i.e. high temperature, MR elastomer made of natural rubber was used in the design. In this design, four MR elastomer samples were arranged to hold up the mount in the diagonal direction similar as in the layout of conventional differential mount. Four coils were used to generate magnetic field to effectively energize MR elastomers in the device. The field directions are shown in figure 22.

\subsubsection{Base isolators for civil engineering application}

Base isolation is a particular application of vibration isolation for civil engineering, which is aimed at preventing earthquakes from transmitting their damage energy into the protected structures, figure 23. Unlike vibration absorbers and vibration isolators mentioned earlier, base isolator requires flexible lateral stiffness while maintains high stiffness vertically to carry large vertical loading due to structural weights. Elastomeric bearings (or rubber bearing) containing multi-layers of rubber material and metal sheets normally form base isolation system for this purpose. As being a passive type device, traditional rubber bearings are vulnerable during earthquakes since they are designed to cope with earthquakes of particular characteristics [78]. Being adaptive for wide range of earthquakes is of great importance due to the versatile nature of the earthquake. Detailed explanation of the vulnerability of traditional base isolation system can be found in [20, 78-79]. 


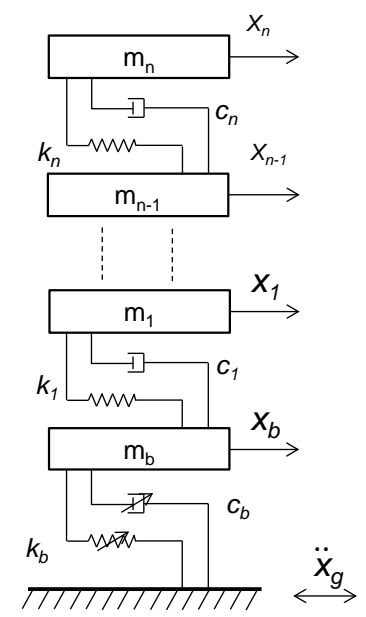

Figure 23. Sketch of adaptive base isolation system featuring MR elastomer for civil structures

To address this issue, several researchers have explored the feasibility in using MR elastomer into base isolation system of civil structures. Hwang et al [118] and Usman et al [119] explored the feasibility through numerical simulations. Numerical results show that base isolation system with MR elastomer outperforms the conventional system in reducing the responses of the structures during seismic excitations. Jung et al [92] built a small scale testing with single floor structure sitting on top of MR elastomer and conducted experimental testing for the simplified system under sinusoidal loading and artificial earthquakes. Behrooz et al $[93,111]$ proposed a variable stiffness and damping isolator to be used in base isolation system of civil structure, figure 24. Four trapezoid MR elastomer samples, with $12 \mathrm{~mm}$ thick, were used in the design with shim separated them. Each two MR elastomer samples form a group arranged as shown in the figure. Four coils on top and bottom of the samples are used to supply magnetic field. Coils were caped within two steel caps together with steel cores. Overall size of the isolator is $128 \mathrm{~mm}$ x $64 \mathrm{~mm} \times 110 \mathrm{~mm}$. Numbers of each coil are 800 and power requirement of each device is $234.2 \mathrm{~W}$ at $4 \mathrm{~A}$. Shear performance of the device under excitation with amplitude of $1 \mathrm{~mm}$ and frequency of $0.1 \mathrm{~Hz}$ is shown in figure 25 . An increase of $57 \%$ was found within the loops. However, major concern for this design for seismic protection of civil structures is the limited vertical loading capacity.

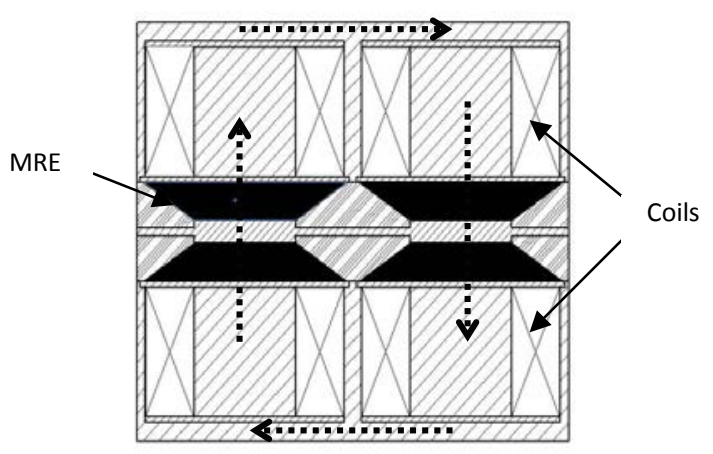

(a)

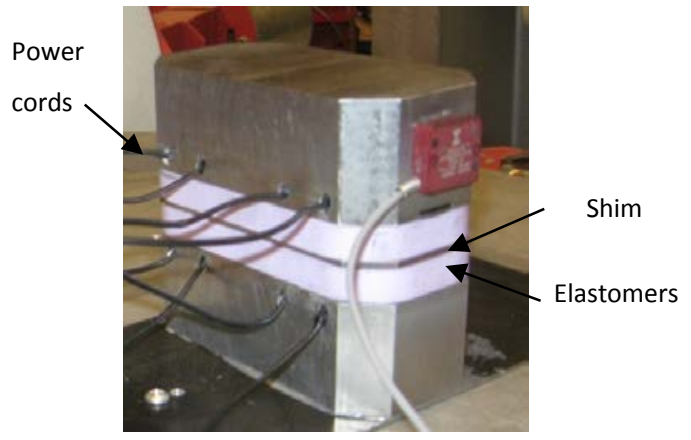

(b)

Figure 24. Variable stiffness and damping isolator proposed by Behrooz et al [93, 111]: (a) cross section view; and (b) prototype. 


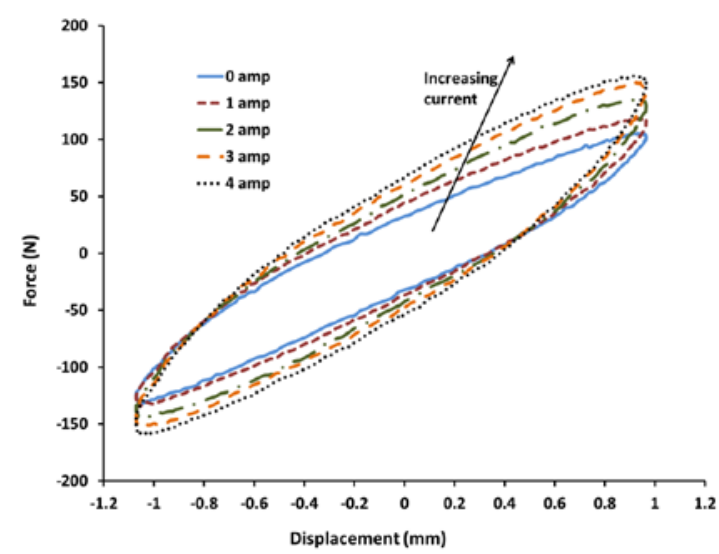

Figure 25. Shear deformation test result of the variable stiffness and damping isolator by Behrooz et al [93, 111] at $0.1 \mathrm{~Hz}$

$\mathrm{Li}$ et al [78-79] proposed the first laminated base isolator containing 47 layers of $\mathrm{MR}$ elastomer sheets with thickness of $2 \mathrm{~mm}$ each and diameter of $140 \mathrm{~mm}$, figure 26 . Between the MR elastomer layers are 46 steel sheets with same diameter and thickness of $1 \mathrm{~mm}$. Such design makes the laminated structure having total height of $140 \mathrm{~mm}$, being the largest and thickest so far. A hollow cylindrical coil was placed outside of the laminated structure. Steel plates at two ends, hollow cylindrical steel yoke, cylindrical steel block and the laminated structure form an enclosed magnetic path. Two cylindrical steel blocks together with laminated structure are used to serve as the core of the magnetic coil. Advantages of laminated design are: (1) large loading capacity of the device; (2) improved magnetic conductivity of the structure; and (3) widely accepted in base isolator design in practice. This device has estimated vertical loading capacity of $370 \mathrm{~kg}$ at the weakest case, i.e. no magnetic field applied and at maximum shear displacement of $26 \mathrm{~mm}$. It is expected to have even larger loading capacity with magnetic field applied and smaller deformations. Such large loading capacity fits to the requirement for applications in seismic protection of civil structures. Experimental testing using shake table demonstrated that this device has 37\% increase on effective stiffness and 45\% increase of maximum force under an applied current of $5 \mathrm{~A}$, figure 27. Uniformed magnetic field of $0.3 \mathrm{~T}$ is estimated to energize all $\mathrm{MR}$ elastomer layers in the device.

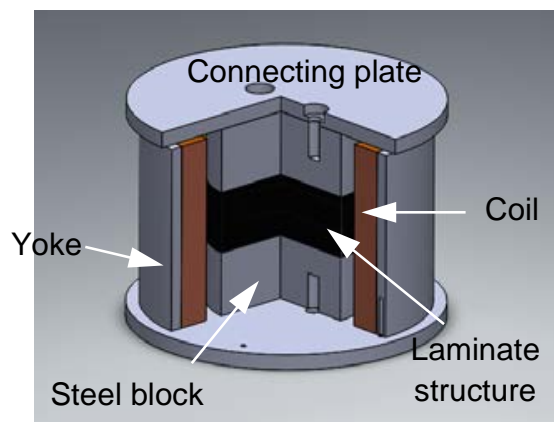

(a)

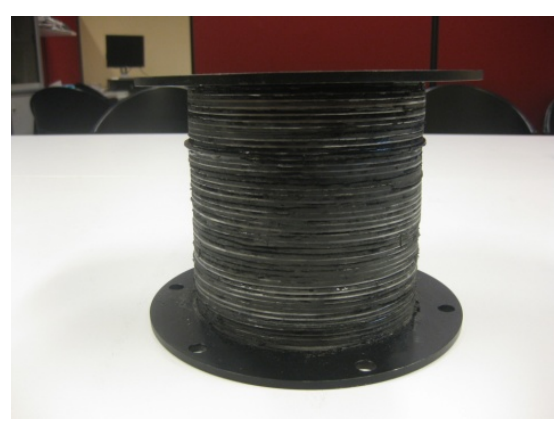

(b)

Figure 26. Laminated MR elastomer base isolator designed by Li et al [78-79]: (a) cross section view of MR elastomer base isolator; and (b) laminated MR elastomer and steel structure 


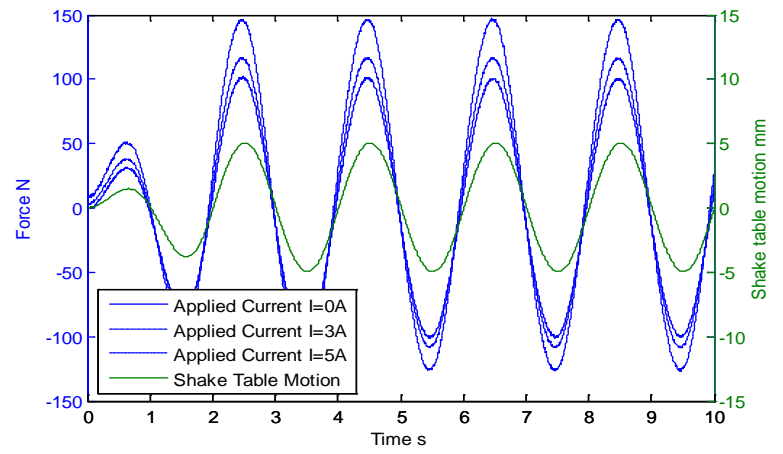

(a)

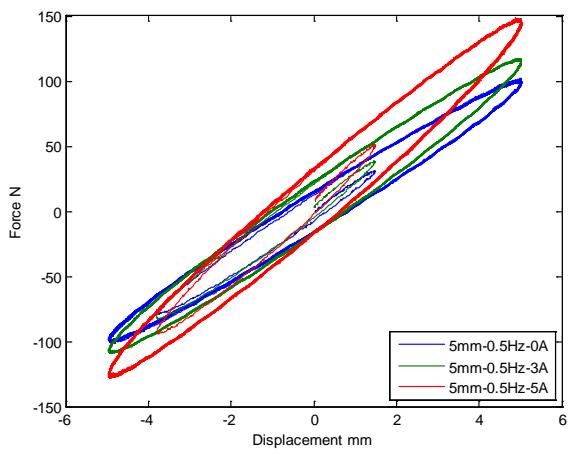

(b)

Figure 27. Performance of the laminated MR elastomer base isolator designed by Li et al [78]: (a) time history of the force record: (b) force-displacement loops at $5 \mathrm{~mm}$ and $0.5 \mathrm{~Hz}$ sinusoidal excitations.

To carry further research, a high adjustable laminated MR elastomer base isolator was designed and tested by $\mathrm{Li}$ et al [20] using soft MR elastomer. MR elastomer used in the new device exhibits an increase of more than 13 folds in shear modulus when subjected to a magnetic field of $0.44 \mathrm{~T}$. The device contains 25 layers of MR elastomer sheets with thickness of $1 \mathrm{~mm}$ and diameter of $120 \mathrm{~mm}$. With the applied current of $3 \mathrm{~A}$, the device possesses great increases of more than 16 folds on shear modulus and more than 14 folds in maximum shear force. Force-displacement hysteresis loops of the device are shown in figure 28. Besides large adaptive range of the device, one of the important observations from this work is the reporting of strain-stiffening behavior, figure 28. It refers to the increasing stiffness in single force-displacement loop at large shear deformations. This behavior has been reported to associate with passive elastomeric bearings as its feature. However, for MR elastomer device, it is the first case.

The work by Li et al [20, 78-79] has proved a feasible solution in using laminated structure for MR elastomer device and effectiveness of the magnetic design. Eem et al [88] has also tempted to design a base isolator $(25 \mathrm{~mm} \times 25 \mathrm{~mm} \times 19 \mathrm{~mm}$ ) with four aluminum plates inserted in the MR elastomer compound. However, such small laminated structure requires two very large electromagnetic coils placed on its top and bottom. Application of such design in seismic base isolation is unlikely.

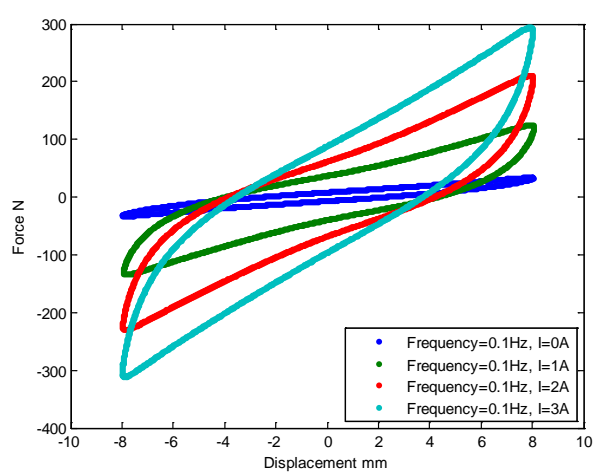

(a)

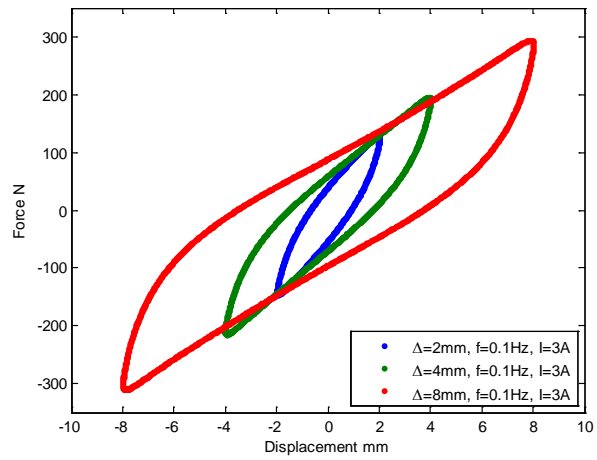

(b)

Figure 28. Performances of highly adjustable laminated MR elastomer base isolator designed by Li et al [20]: (a) forcedisplacement loops at $8 \mathrm{~mm}$ and $0.1 \mathrm{~Hz}$ cyclic loading at different currents; (2) force-displacement loops at different amplitude (2mm, $4 \mathrm{~mm}$ and $8 \mathrm{~mm}$ ) excitation at $0.1 \mathrm{~Hz}$ and $3 \mathrm{~A}$. 


\subsection{Sensing devices}

Besides the field-sensitive elastic property, MR elastomers (particularly the anisotropic form) possess several functions such as magnetoelasticity, magnetoresistance, magnetostriction, piezoeresistance and theromoresistance [109]. Reasons behind these are the changes in the spacing between particles along chain structures due to external loadings which produces variation of electrical property of the material. With such properties, they have potentials to develop sensing devices, such as temperature, pressure and chemical sensors. Ginder et al [27] discovered that the electric constant and conductance of MR elastomer decrease when the material is subjected to external loading, i.e. shear or stretch. Martin et al [120] reported an extremely large dependence of effective conductivity of MR elastomer on strain or elastic deformation. Brownian dynamic simulation was used to explain the magnetostriction effect of the material. They also discovered that the magnetostriction of anisotropic MR elastomer is highly dependent on the structure of the particle agglomerates [121]. Wang et al [122] utilized Impendence Spectroscopy technology to investigate the electric response of MR elastomers under combined influences of deformation and magnetic field. A phenomenological model was proposed to understand its impendence response. Kchit and Bossis [47-48, 123] comparatively studied the electrical resistivity of metal powers, anisotropic MR elastomers and isotropic MR elastomers. It is revealed that the change in resistance in compression under pressure for anisotropic MR elastomer is 10 times larger than that of isotropic MR elastomer with same volume fraction of iron particles. Tian et al [124] experimentally evaluated the sensing capacities of eight MR elastomer samples with different graphite weight fractions. Theoretical analysis based on dipole model was conducted to explain the experimental findings.

To date, research and developments on MR elastomer based sensing devices is rarely found. A force sensor working with MR elastomer was designed by Li et al [73], shown in figure 29. The force sensor is designed to identify the normal loading. Experimental testing showed its good linear relationship and repeatability between the voltage output and loading force. Bica [125] designed a magnetoresisteor sensor using graphite MR elastomers with aim to detect the magnetic field. Du and Chen [126] prototyped a small scale MEMS magnetometer based on MR elastomer. This device has good linearity within some range of magnetic field, i.e. 0$120 \mathrm{kA} / \mathrm{m}$.

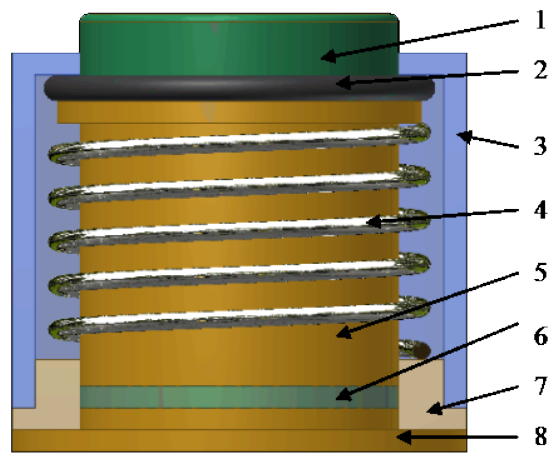

(a)

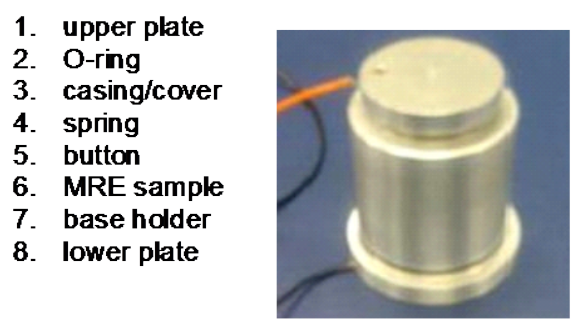

(b)

Figure 29. (a) Schematic design of the MRE force sensor; (b) picture of the sensor prototype. [73] 


\subsection{Other MR elastomer devices}

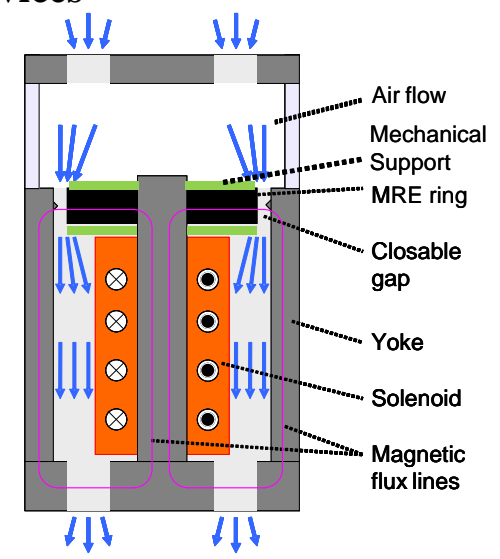

Figure 30. Controllable valve using soft MR elastomer as actuators [75]

Field-active mode (see section 2.1) originates from magnetostrictive behavior of MR elastomers, i.e. the tendency of magnetized particles to move along magnetic field. Noted from [120, 122] that magnetostrictive MR elastomers possess advantages such as a much larger strain and faster response time. Therefore, they can be used for the development of soft actuators, fast artificial muscles and so on. Bose et al [75] designed a controllable valve using soft MR elastomers, shown in figure 30. This device is to regulate air flow through the controllable valve. The MR elastomer ring will expand radially on the application of magnetic field and therefore the closable gap between the yoke and the ring shrinks. Changing the magnetic field will thus adjust the air flow through the closable gap. Kashima et al [77] proposed a soft actuator using isotropic MR elastomer, shown in figure 31. Three parts in the actuator are electromagnetic coil, soft silicon elastomer and MR elastomer cover. The MR elastomer is slight harder than the enclosed soft silicon elastomer. Under magnetic field, energized MR elastomers contract to push the soft elastomer. Original shape recovers after the removal of magnetic field.
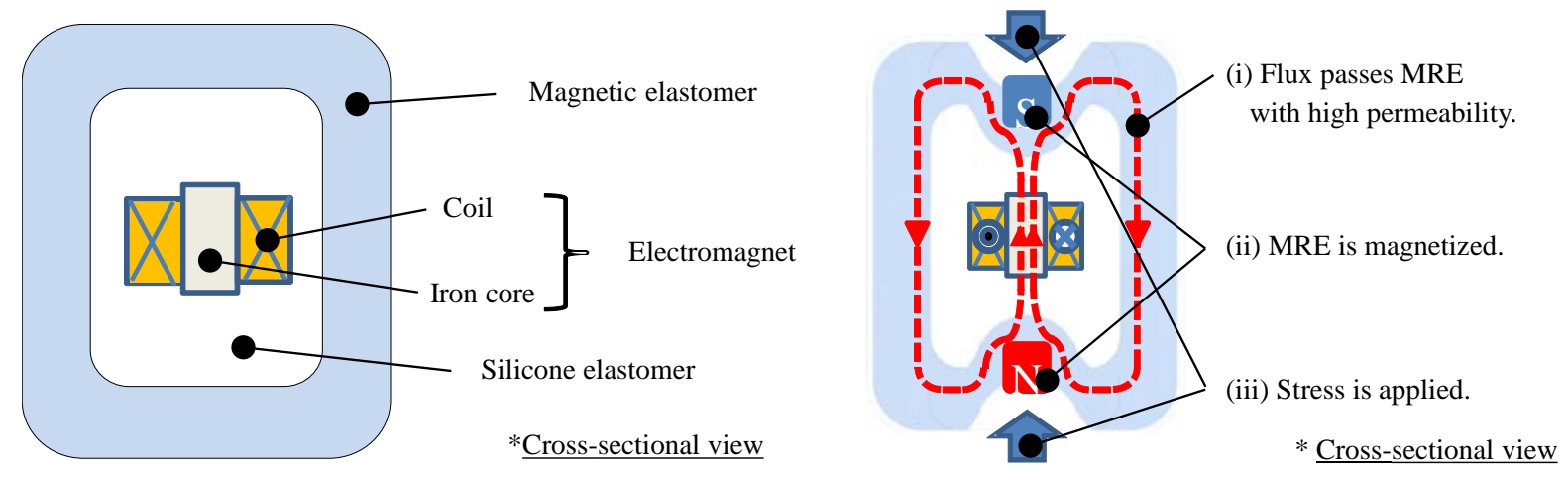

Figure 31. Soft actuator made of isotropic MR elastomer [77]

Physical status shift of MR elastomer due to magnetic field makes it great candidate to develop adaptive beam structures [94, 96-97, 127]. Zhou and co-workers [94, 96-97] piloted the idea of adaptive sandwich beams with MR elastomers as part of the core, figure 32 (a). Extensive numerical simulations and finite element analysis have been conducted. Experimental verifications were conducted by Nayak et al [103] and applying magnetic field realized a significant attenuation of structural vibration. Another sandwich beam design with 
entire core as MR elastomers was proposed by Ying and Ni [82]. Experimental verifications of such design have been conducted by Lara-Prieto et al [101] and Hu et al[128], figure 32 (b).

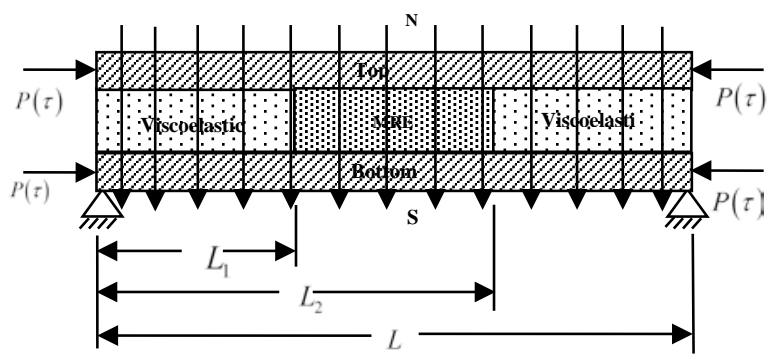

(a)

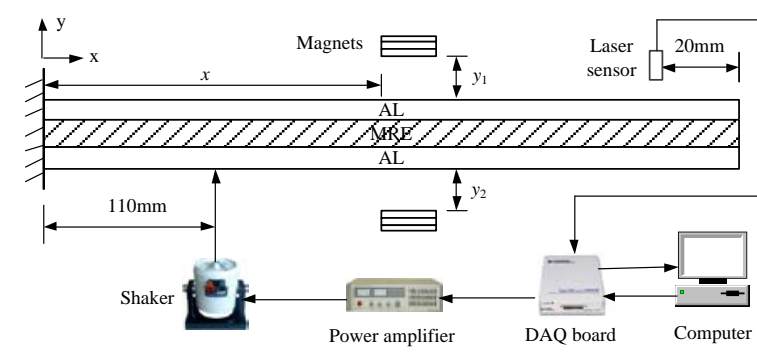

(b)

Figure 32. MR elastomer sandwich beam designs: (a) with MR elastomer as part of the core [103]; (b) with MR elastomer as core [128]

\section{Modeling the mechanical behavior of MR elastomer material and devices}

\subsection{Rheological modeling of MR elastomer material}

MR elastomer is a typical nonlinear viscoelastic material with field-dependent mechanical properties, i.e. stiffness and damping. Modeling of the complex behavior of MR elastomers is an essential step towards its engineering applications [129]. Despite a handful of research on the material properties, modeling of the hysteresis behavior requires more attention. Till now, there is still limited work on the modeling of complex behavior of MR elastomer. Much of the efforts are built on the previous research on the modeling of MR fluids [130-131].

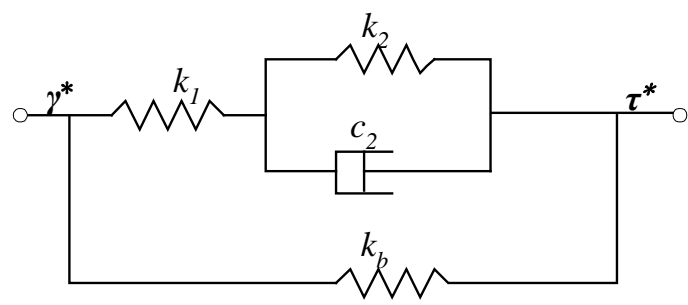

Figure 33. Four-parameter viscoelastic model proposed by Li et al [50]

Three-parameter model is normally used to describe the viscoelastic behavior of rubber material. The three-parameter is a combination of stiffness element and a classical Kevin model (being a stiffness element and damping element in parallel). The additional of stiffness element is to improve the efficiency of Kevin model under sudden loading. Built on this model, Li et al [50] developed a four-parameter viscoelastic model, as shown in figure 33. In this model, a stiffness element is in parallel with the three-parameter model. This model was proven to be effective to describe the behavior of MR elastomer, figure 34. Eem et al [58] proposed a dynamic model with Ramberg-Osgood model and a Maxwell model in parallel, figure 35. The Ramberg-Osgood model is to describe the nonlinearity of the material while the Maxwell model is to introduce viscoelastic element into the model. This model also shows good agreement with experimental data. Chen and Jerrams [53] proposed a rheology model which considers the viscoelasticity of the polymer composite, magnetic field-induced properties and interfacial slippage between the matrix and particles, figure 36 . The viscoelasticity was modeled by standard linear solid model which contains two spring 
elements and a damping element. The field-dependent stiffness property was described by a variable stiffness spring and the interfacial slippage between the matrix and particles was reproduced by a spring-Coulomb friction slider. In addition, Koo et al [49] proposed a model using neural network to reproduce the behavior of MR elastomer under compressive loading.

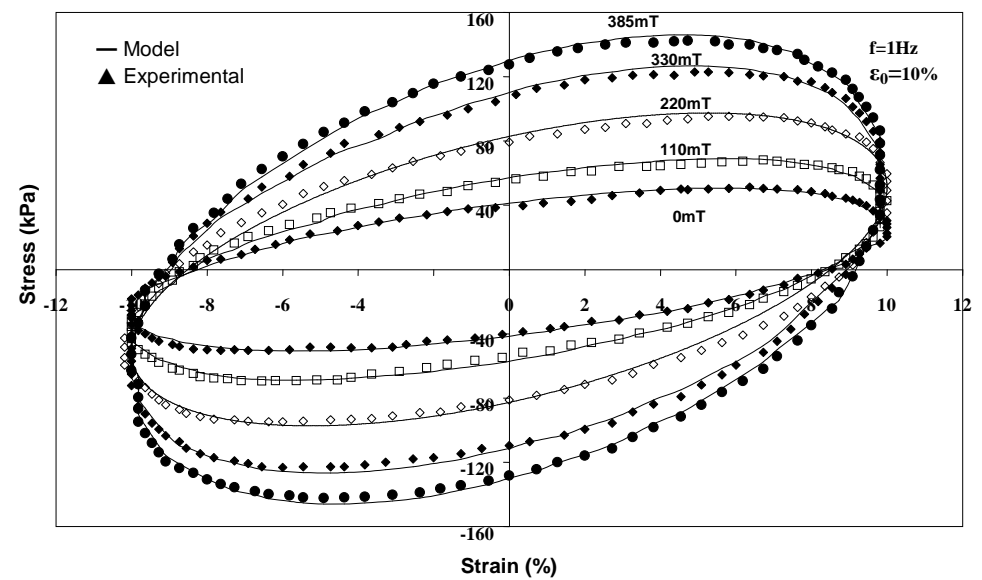

Figure 34. A comparison between experimental data with model-predicted results with the amplitude input of 10\% [50].

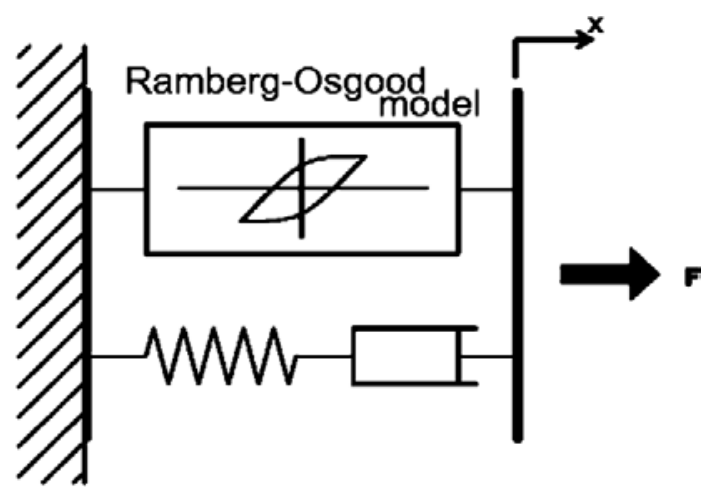

Figure 35. Dynamic model of MR elastomers proposed by Eem et al[58]

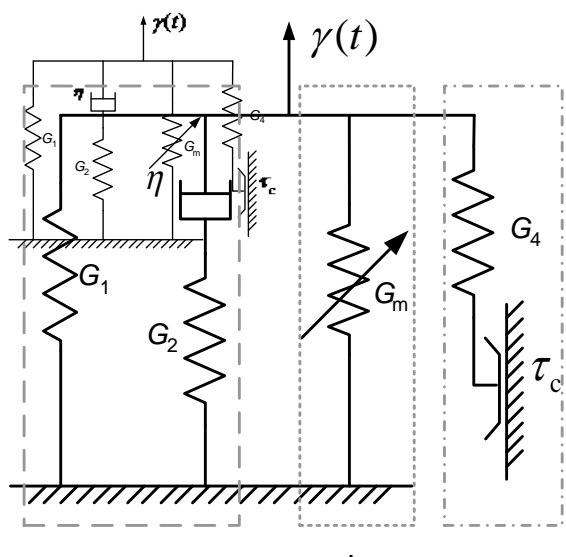

a

b

C

Figure 36. A rheological model proposed by Chen and Jerrams [53] for examining dynamic properties of MR elastomers comprising three components to simulate (a) the viscoelasticity of the polymer composite, (b) the magnetic-field-induced mechanical properties, and (c) interfacial slippage between the matrix and the particles 


\subsection{Phenomenological model of MR elastomer devices}

With hysteresis property from the material medium, MR elastomer devices inevitably possess nonlinear hysteresis, evident by [20, 93,111]. Unlike MR fluid device, MR elastomer devices have been identified with strain-stiffening behavior, shown in figure 28. Because of limited cycling characterization test of MR elastomer devices [20, 78-79, 93,111], very few modeling efforts have been reported till now.

Comprehensive review on models of MR fluid dampers can refer to work done by Wang and Liao [132]. Due to the distinct difference between MR elastomer device and MR fluid damper, it is expected that models for MR fluid damper do not entirely work for MR elastomer devices. Bouc-Wen model, as a classical hysteresis model, can be used or modified to describe the behaviors of MR elastomer base isolators [93, 133], shown in figure 37. Yang et al [133] proposed Bouc-Wen model and explained the impact of different model parameters on hysteresis shape. Behrooz et al [93] modified Bouc-Wen model by adding a standard three-element solid model in parallel. $\mathrm{Li}$ and $\mathrm{Li}$ [134-135] proposed a strainstiffening model for MR elastomer base isolator, figure 38 (a). In this model, the upper branch is the standard three-parameter solid model and the lower branch is a modified Maxwell model which contains a strain-stiffening spring element and a dashpot element connected in series. The strain-stiffening element is a power law function. Parameter optimization of this model has been explored by Yu et al [136-137]. Yu et al [138] proposed a hyperbolic sine function to describe the strain-stiffening behavior associated with MR elastomer base isolator, figure 38 (b). In addition, Dahl model [139] and black box model [140] can also be used for such purpose.

Another difference between MR fluid device and MR elastomer devices during modeling is that the magnetic field in MR elastomer device may vary with their motions. Li and Li [135, 141] discovered that the deformation of the MR elastomer changes the magnetic field distribution and therefore magnitude of the magnetic field for MR elastomer base isolator, figures 39 and 40. This study is based on the MR elastomer base isolator in [20]. Finite element analysis was conducted for various horizontal deformations, i.e. $0 \mathrm{~mm}, 5 \mathrm{~mm}, 10 \mathrm{~mm}$ and $15 \mathrm{~mm}$. It can be found that the magnetic field decreases along the deformation of the device, figure 40. Due to the independence of magnetic field with motion, magnetic field in MR fluid devices is considered only related to applied current during modeling process. However, for MR elastomer devices, it may not be the case. It is expected that for squeeze/elongation mode MR elastomer device, variation of the magnetic field due to motion is more obvious. Therefore, finite element analysis should be undertaken before the modeling taking place for MR elastomer devices. 


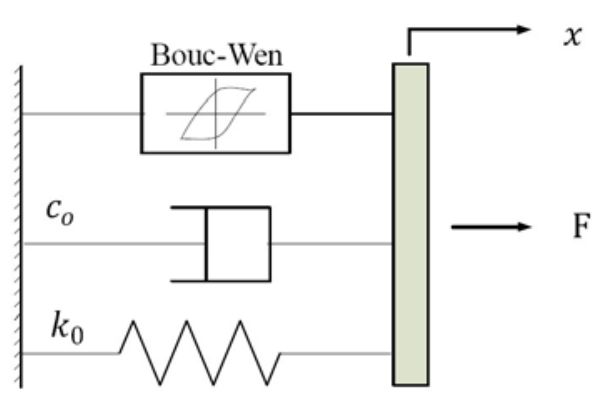

(a)

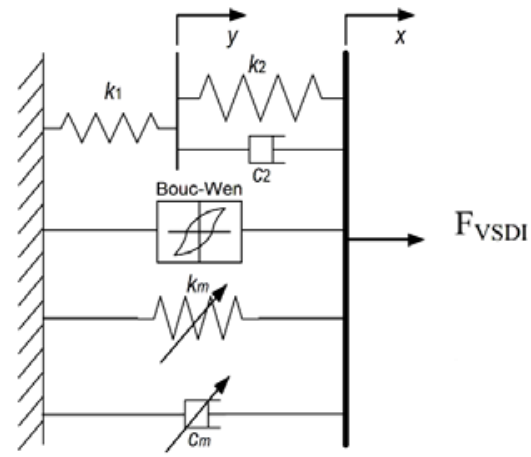

(b)

Figure 37. Bouc-Wen models for MR elastomer base isolators: (a) model proposed by Yang et al [133]; [b] model proposed by Behrooz et al [93].

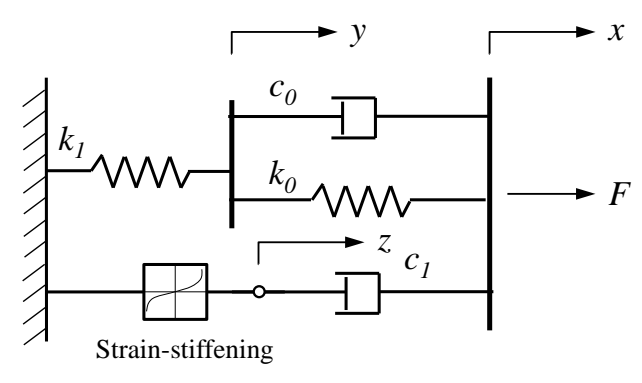

(a)

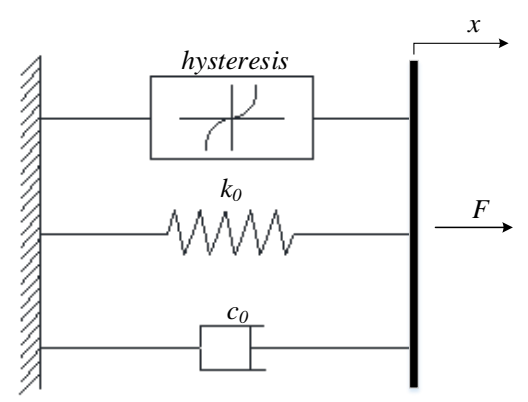

(b)

Figure 38. Models for MR elastomer base isolators: (a) strain-stiffening model proposed by Li and Li [134]; (b) hyperbolic model proposed by Yu et al [138].

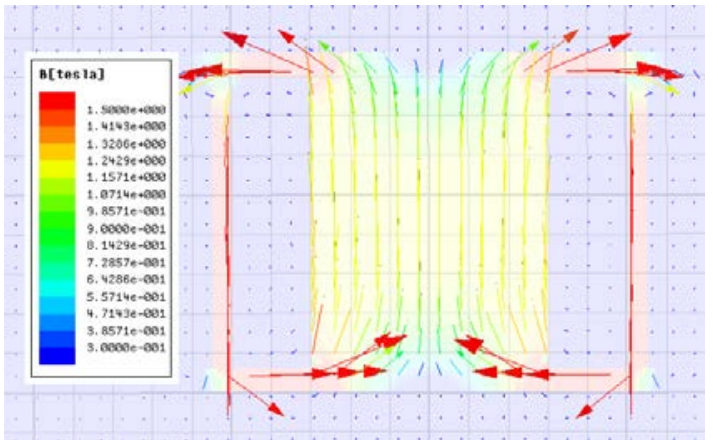

(a)

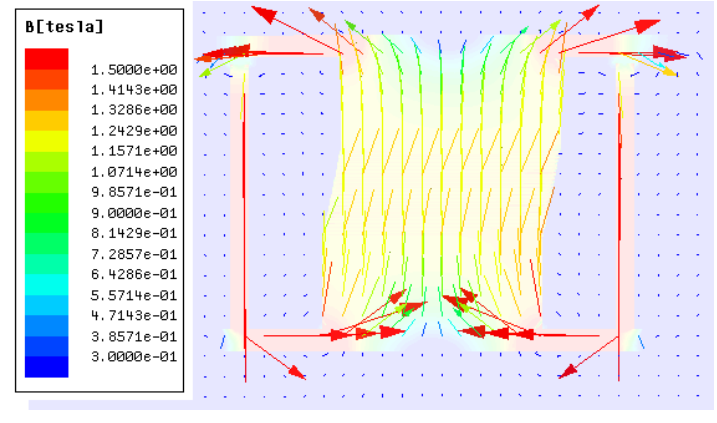

(c)

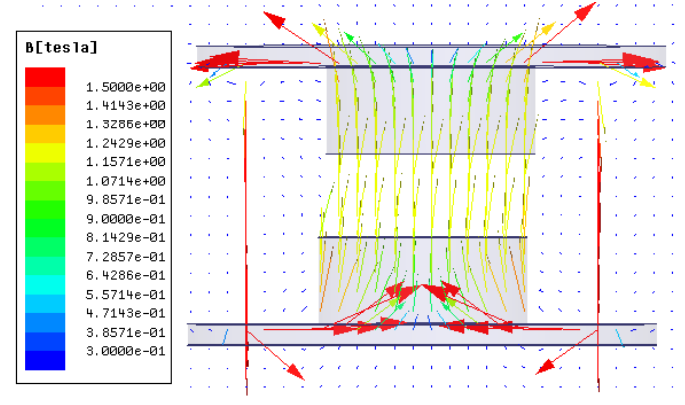

(b)

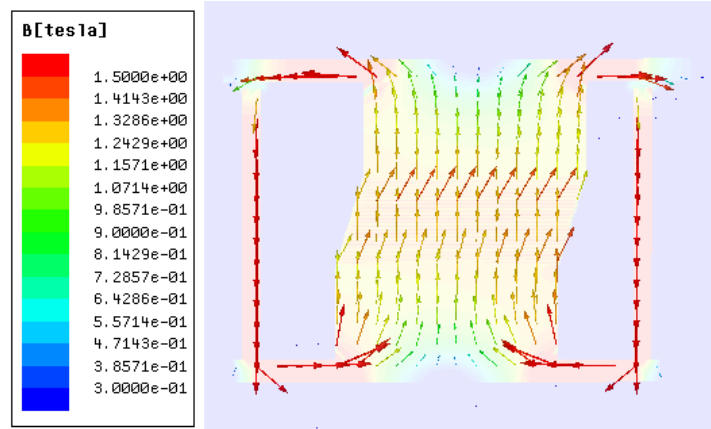

(d)

Figure 39. Magnetic flux line and magnitude in MRE base isolator [141]: (a) no deformation; (b) 5mm deformation; (c) $10 \mathrm{~mm}$ deformation and (d) $15 \mathrm{~mm}$ deformation; 


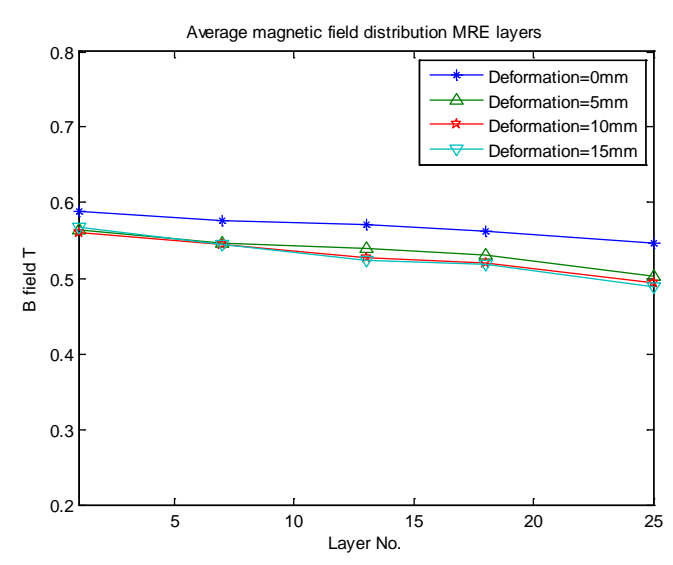

Figure 40. Average magnetic field in MRE layers for various deformation (I=0.5A)

\section{Opportunities and challenges}

In last decades, research development on MR elastomer has been blooming with numerous research activities ongoing in every aspect of related fields from material science to engineering applications. Increasingly understanding on the material property itself and innovative design of MR elastomer devices have brought opportunities for the application of MR elastomer in various engineering fields. Yet, new developments are on the way and we certainly have not fully explored its potentials. In addition, endorsements from the material property and demands from practical engineering applications oppose challenges which require attentions to be addressed. Some of these challenges are associated with material itself while much of these are bound up with innovative thinking and design.

Applications of MR elastomer require comprehensive understanding on the material property. Properties of MR elastomers under tension, shear deformation and compression have been investigated by a number of researchers [3, 41, 49-51, 58, 129]. In engineering applications of MR elastomer, such as vibration isolation and absorption, the materials mostly are subjected to combined loadings, for example, combined lateral shear and compressive loadings. Research on combined loading conditions is still limited and extra efforts are required to meet needs of applications.

Invention of MR elastomers provides potential solutions to many issues in MR fluids, such as particle settling, sealing problem and environmental contamination etc. Explorations on use of MR elastomer till now have been mainly limited on applications of vibration reduction and isolation. Expectations of its potential applications may include many, if not all, of the discovered applications of MR fluids, such as braking system, haptic devices and so on. Among three operation modes, shear mode has been well studied. Yet MR elastomer devices with combined working modes should be further explored. Exploring new operation mode for MR elastomer is another task to expand its engineering applications. In addition, with the sensing capacities when subjected to external loading, self-sensing MR elastomer devices should be a potential research topic, which offers a great value for an integrated control system design with real-time feedback of the device status.

General requirements for development of MR elastomer devices include large adaptive working frequency range, low power consumption and compact configuration. Devices with 
large MR effect (i.e. large change in their modulus and damping) between field-on and fieldoff status can potentially provide large adaptive range, given that sufficient magnetic field is provided. It all boils down to the development of high performance MR elastomer material. Three factors affecting the properties of MR elastomer are the volume fraction of iron particles, magnetic particle size and base matrix. Optimal volume fraction of the iron particle has been found to be around $27 \%$. While using soft matrix material can produce large MR effect, however, MR elastomer with soft rubber matrix requires much longer vulcanizing time [20] and will reduce the loading capacity as well as durability of the device, which hinders its applications for cases where such attributes are requires. Therefore, trade-off between high MR effects and other performance criteria of MR elastomer should be carefully considered. Wider particle size has reported to enhance the MR effect which is one of possible options to develop high performance material [11].

Most of MR elastomer devices reported so far require relative large power consumption, from tens watts to hundreds watts, and have large device size due to existence of conductive magnetic path. These factors may limit engineering applications for MR elastomer devices where required power supply may not be feasible or sustainable and allowable space may be constrained. The cause of such drawback of MR elastomer device is that usually thick MR elastomers with considerable size (consequently over-size devices) are placed in the devices to meet the performance requirements. Fully energizing these materials requires strong magnetic flux produced by large coils with high electrical resistance. Possible solution for the problem may be found by the optimal design of the magnetic circuit, such as coil placement, where extensive numerical analysis can be applied to gain insight on how to minimize the energy requirement. However, due to lack of the data on B-H curve of MR elastomer, numerical analysis cannot be accurately conducted. If sufficient information was available, the design of compact MR elastomer devices with lower energy consumption could be achieved with help of numerical simulation in design.

Large-scale MR elastomer devices have rarely reported due to the need for involvement of large size MR elastomer. Low permeability of MR elastomer makes it a challenge task to provide strong and uniform magnetic field to the material. By far, the device with largest MR elastomer involved is MR elastomer base isolators $[20,78]$ where MR elastomer samples are with diameter over $100 \mathrm{~mm}$ and thickness of $94 \mathrm{~mm}$ and $25 \mathrm{~mm}$, respectively. In addition, these devices also possess large load-carrying capacity which is not found elsewhere. This is due to the adoption of laminated magnetic core which composes alternative MR elastomer and steel layers. In engineering applications, large size and loading capacity are two of the major concerns facing MR elastomer devices. Developments towards these objectives need further exploration.

Understanding the complex behavior of MR elastomer devices is critical to their future development and applications. To start, standard characterization tests, i.e. static test and cycling test, should be conducted for all devices. However, research on this aspect has been mainly focused on the frequency shifting capacity of the devices, such as for vibration absorbers and vibration isolators. Only few experimental investigations on their cycling behaviors have been reported [20, 78-79, 93,111]. As such, efforts in developments of 
mathematical models are also limited. A well understood and accurately modeled MR elastomer device is capable of taking full advantages of its unique feature in its application such as design of device control etc.

Another challenge on the potential applications of MR elastomer devices is to develop appropriate control strategies for the various applications to utilize their uniqueness and potentials. For example, one of the effective ways of vibration mitigation is to shift fundamental frequency of the system away from the harmful resonance frequency. One the other hand, increasing damping in the system can also suppress vibration by dissipating vibration energy but has little impact on natural frequency of the structure. The recent development of MR elastomer devices makes MR elastomer based variable stiffness system feasible, since the highly adjustable stiffness of MR elastomer devices enables significant frequency shift of the system. This is particularly attractive for vibration isolation applications, as in vibration isolation systems, the primary objective for designing a control algorithm is to shift the natural frequency as far as possible from the excitation frequency. Varying stiffness based on the prediction or preview of the frequency range of the possible vibration sources could be an effective way. However, to identify upcoming frequency characteristics of the vibration sources for real-time control is a challenge since it requires certain period of feedback accumulation.

Application of MR elastomer technology in civil engineering structures, especially for base isolation system, has attracted increasing attentions recently. Adaptive base isolators made of MR elastomer has been successfully designed and tested [20]. Though several small scale testing have been conducted, i.e. on a single-story building with overall building mass of 15 $\mathrm{kg}$ [92] and on a two-story building with overall building mass of $68 \mathrm{~kg}$ [111], behaviors of civil structures under earthquakes is much more complicated. Besides, experimental verifications on civil structures should involve benchmark buildings approved by international associations thus the results can be accepted by the community. Several case studies on the control development with a five-storey benchmark building model have been conducted [142-144]. Further experimental validation is on the way to explore the application of MR elastomer in base isolation of civil structures.

Opportunities emerge with challenges along the research advances on MR elastomer technology. With great potentials of MR elastomers in engineering applications, resolving the challenges leads to improvement and new development in the field. As technology matures gradually, MR elastomer convincingly will be applied and shape the outlook of many engineering applications.

\section{Acknowledgement:}

The authors would like to acknowledge the support from colleagues to provide original figures for the article. Kind support from Dr John M. Ginder at Ford Corporation, Prof. Xinglong Gong at University of Science and Technology of China, Prof. Faramarz Gordaninejad at University of Nevada, Reno, USA, Prof. Ken Curefare at Georgia Institute of Technology, USA, Prof. S. K. Dwivedy at Indian Institute of Technology, Guwahati, India, Prof. Kyong-Soo Yim and Dr Young-Keun Kim at Korea Advanced Institute of Science and 
Technology, Prof. Holger Böse at Fraunhofer-Institut für Silicatforschung ISC, Germany, Dr Barkan Kavlicoglu at Advanced Materials and Devices Inc., Nevada, USA, Prof. Katsuhiro Hirata and Dr. Shunta Kashima at Osaka University, Japan, Dr Lin Chen from the Commercial Aircraft Corporation of China, Prof. Yim Woosoon from University of Nevada, Las Vegas and Dr Saul Opie from Boeing, are greatly appreciated.

\section{References:}

[1]. Carlson J D and Jolly M R 2000 MR fluid, foam and elastomer devices Mechatronics 10 555-69

[2]. Chen L, Gong X and Li W 2007 Microstructures and viscoelastic properties of anisotropic magnetorheological elastomers Smart Mater. Struct. 16 2645-49.

[3]. Popp K, Kroger M, Li W, Zhang X and Kosasih P 2010 MRE Properties under shear and squeeze modes and applications J. Intell. Mater. Syst. Struct. 21 1471-1477.

[4]. Rabinow J 1948 Magnetic Fluid Clutch National Bureau of Standards Technical News Bulletin 32 54-60

[5]. Rabinow J 1951 Magnetic Fluid Torque and Force Transmitting Device U.S. Patent 2,575,360.

[6]. Rigbi Z and Jilken L 1983 The response of an elastomer filled with soft ferrite to mechanical and magnetic influences J. Magnetism Magnetic Mater. 37 267-76

[7]. Shiga T, Okada A and Kurauchi T 1993 Elastroviscoelastic effect of a polymer blends consisting of silicon elastomer and semiconducting polymer particles Macromolecules 26 6958-63

[8]. Jolly M R, Carlson J D, Munoz B C and Bullions T A 1996 The magnetoviscoelastic response of elastomer composites consisting of ferrous particles embedded in a polymer matrix J. Intell. Mater. Syst. Struct. 7 613-22

[9]. Kallio M 2005 The elastic and damping properties of magnetorheological elastomers VTT publications No. 565 146p

[10]. Wu J, Gong X, Fan Y and Xia H 2010 Anisotropic polyurethane magnetorheological elastomer prepared through in situ polycondensation under a magnetic field Smart Mater. Struct. 19105007

[11]. Stepanov G V, Abramchuk S S, Grishin D A, Nikitin LV, Kramarenko E Y and Khokhlov A R 2007 Effect of a homogeneous magnetic field on the viscoelastic behavior of magnetic elastomers Polymer $\mathbf{4 8}$ 488-95

[12]. Leblanc J L 2002 Rubber-filler interactions and rheological properties in filled compounds Progress in Polymer Sci. 27 627-687.

[13]. Munoz B C and Jolly M R 2001 Composites with field responsive rheology Performance of Plastics Munich Carl Hanser Verlag pp: 553-74

[14]. Lokander M and Stenberg B 2003 Performance of isotropic magnetorheological rubber materials Polymer Testing 22 245-51.

[15]. Tian T F, Li W H, Alici G, Du H and M Y Deng 2011 Microstructure and magnetorheology of graphitebased MR elastomers Rheol. Acta 50 825-836.

[16]. Liao G J, Gong X L, Xuan S H, Kang C J and Zong L H 2012 Development of a real-time tunable stiffness and damping vibration isolator based on magnetorheological elastomer J. Intell. Mater. Syst. Struct.23 25-33

[17]. Li W H, Zhang X Z and Du H 2013 Magnetorheological elastomers and their applications Adv. Struct. Mater. 11 357-74

[18]. Shen Y, Golnaraghi M F and Heppler G R 2004 Experimental research and modeling of magnetorheological elastomers J. Intell. Mater. Syst. Struct.15 27-35

[19]. Deng H X, Gong X L and Wang L H 2006 Development of an adaptive tuned vibration absorber with magnetorheological elastomer Smart Mater. Struct. 15 N111-16

[20]. Li Y, Li J, Tian T, Li W 2013 A highly adjustable magnetorheological elastomer base isolator for applications of real-time adaptive control Smart Mater. Struct. 22095020.

[21]. Padalka O, Song H J, Wereley N M, Filer J A and Bell R C 2010 Stiffness and damping in Fe, Co, and Ni nanowire-based magnetorheological elastomeric composites IEEE Trans. Magnet. 46 2275-77

[22]. Kallio M, Lindroos T, Aalto S, Jarvinen E, Karna T and Meinander T 2007 Dynamic compression testing of a tunable spring element consisting of a magnetorheological elastomer Smart Mater. Struct. 16 506-14 
[23]. Jolly M R, Carlson J D and Munoz B C 1996 A model of the behaviour of magnetorheological materials Smart Mater. Struct.5 607-14

[24]. Zhou G Y 2003 Shear properties of a magnetorheological elastomer Smart Mater. Struct. 12 139-46

[25]. Zhou G Y and Li J R 2003 Dynamic behavior of a magnetorheological elastomer under uniaxial deformation: I. Experiment Smart Mater. Struct. 12 859-72

[26]. Ginder J M, Nichols M E, Elie L D and Tardiff J L 1999 Magnetorheological elastomers: properties and applications Smart Structures and Materials 1999: Smart Materials Technologies, 131-138, 1999.

[27]. Ginder J M, Nichols M E, Elie L D and Clark S M 2000 Controllable-stiffness components based on magnetorheological elastomers Smart Structures and Material 2000: Smart Structures and Integrated Systems, Proceedings of SPIE 3985, 418-25.

[28]. Chen L and Gong X L 2008 Damping of magnetorheological elastomers J. Central South Uni. 15 271-74

[29]. Fan Y, Gong X L, Xuan S, Zhang W, Zheng J and Jiang W Q 2011 Interfacial friction damping properties in magnetorheological elastomers Smart Mater. Struct.20 035007

[30]. Ginder J M, Scholotter W F and Nichols M E 2001 Magnetorheological elastomers in tunable vibration absorbers Smart Structures and Materials 2001: Damping and Isolation, Proceedings of SPIE 4331 10310

[31]. Davis L C 1999 Model of magnetorheological elastomers J. App. Phys. 85 3348-51

[32]. Borcea L and Bruno O 2001 On the magneto-elastic properties of elastomer-ferromagnet composites $J$. Mech. Phys. Solids 49 2877-919

[33]. Yin H M, Sun L Z and Chen J S 2006 Magneto-elastic modeling of composites containing chainstructured magnetostrictive particles J. Mech. Phys. Solids 54 975-1003

[34]. Zhang X Z, Li W H and Gong X L 2008 An effective permeability model to predict field-dependent modulus of magnetorheological elastomers Commun. Nonlinear Sci. Numer. Simul. 13 1910-16

[35]. Yu M, Xia Y Q and Yan X R 2009 Analysis and verification on the chain-like model with normal distribution of magnetorheological elastomer Chin. J. Chem. Phys. 22 545-50

[36]. Guan X C, Dong X F and Ou J P 2009 Predicting performance of polymer-bonded Terfenol-D composites under different magnetic fields J. Magnetism Magnetic Mater. 321 2742 48

[37]. Melenev P, Raikher Y, Stepanov G, Rusakov V and Polygalova L 2011 Modeling of the field-induced plasticity of soft magnetic elastomers J. Intell. Mater. Syst. Struct. 22 531-38

[38]. Danas K, Kankanala SV and Triantafyllidis N 2012 Experiments and modeling of iron-particle-filled magnetorheological elastomers J. Mech. Phys. Solids 60 120-38

[39]. Galipeaua E and Castaneda P P 2013 A finite-strain constitutive model for magnetorheological elastomers: Magnetic torques and fiber rotations J. Mech. Phys. Solids 61 1065-90

[40]. Zhang X Z, Li W H and Gong X L 2008 An effective permeability model to predict field-dependent modulus of magnetorheological elastomers Commun. Nonlin. Sci. Numer. Simul. 13 1910-16

[41]. Demchuk S A and Kuz'min V A 2002 Viscoelastic properties of magnetorheological elastomers in the regime of dynamic deformation J. Eng. Phys. Thermophysics 75 396-400

[42]. Zhou G Y and Jiang Z Y 2004 Deformation in magnetorheological elastomer and elastomer-ferromagnet composite driven by a magnetic field Smart Mater. Struct.13 309-16

[43]. Abramchuk S S, Grishin D A, Kramarenko E Y, Stepanov G V and Khokhlov A R 2006 Effect of a homogeneous magnetic field on the mechanical behavior of soft magnetic elastomers under compression Polymer Sci. Ser. A 48 138-45

[44]. Wang Y, Hu Y, Chen L, Gong X, Jiang W, Zhang P and Chen Z 2006 Effects of rubber/magnetic particle interactions on the performance of magnetorheological elastomers Polymer Testing 25 262-267

[45]. Chen L, Gong X L and Li W H 2007 Microstructures and viscoelastic properties of anisotropic magnetorheological elastomers Smart Mater. Struct. 16 2645-50

[46]. Meunier L, Chagnon G, Favier D, Orgéas L and Vacher P 2008 Mechanical experimental characterisation and numerical modelling of an unfilled silicone rubber Polymer Testing 27 765-777.

[47]. Kchit N, Lancon P and Bossis G 2009 Thermoresistance and giant magnetoresistance of magnetorheological elastomers J. Phys. D: Appl. Phys. 42105506

[48]. Kchit N and Bossis G 2009 Electrical resistivity mechanism in magnetorheological elastomer J. Phys. D: Appl. Phys. 42105505 
[49]. Koo J H, Khan F, Jang D D and Jung H J 2010 Dynamic characterization and modeling of magnetorheological elastomers under compressive loadings 19117002

[50]. Li W H, Zhou Y and Tian T F 2010 Viscoelastic properties of MR elastomers under harmonic loading Rheologica Acta 49 733-40

[51]. Zhang W, Gong X L, Sun T L, Fan Y C, Jiang W Q 2010 Effect of cyclic deformation on magnetorheological elastomers Chin. J. Chem. Phys. 23 226-30

[52]. Zhang W, Gong X L, Jiang W Q and Fan Y C 2010 Investigation of the durability of anisotropic magnetorheological elastomers based on mixed rubber Smart Mater. Struct. 19085008

[53]. Chen L and Jerrams S 2011 A rheological model of the dynamic behavior of magnetorheological elastomers J. App. Phys. 110013513

[54]. Gong X, Liao G and Xuan S 2012 Full-field deformation of magnetorheological elastomer under uniform magnetic field App. Phys. Letter 100211909

[55]. Gordaninejad F, Wang X and Mysore P 2012 Behavior of thick magnetorheological elastomers J. Intell. Mater. Syst. Struct.23 1033-39

[56]. Koo J H, Dawson A and Jung H J 2012 Characterization of actuation properties of magnetorheological elastomers with embedded hard magnetic particles J. Intell. Mater. Syst. Struct.23 1049-54

[57]. Ju B X, Yu M, Fu J, Yang Q, Liu X Q and Zheng X 2012 A novel porous magnetorheological elastomer: preparation and evaluation Smart Mater. Struct. 21035001

[58]. Eem S-H, Jung H-J and Koo J-H 2012 Modeling of magneto-rheological elastomers for harmonic shear deformation IEEE Trans. Magnetics 48 3080-83

[59]. Qiao X Y, Lu X S, Li W H, Chen J, Gong X L, Yang T, Li W, Sun K and Chen X D 2012 Microstructure and magnetorheological properties of the thermoplastic magnetorheological elastomer composites containing modified carbonyl iron particles and poly(styrene-b-ethyleneethylenepropylene-b-styrene) matrix Smart Mater. Struct. 21115028

[60]. Li W H and Nakano M 2013 Fabrication and characterization of PDMS based magnetorheological elastomers Smart Mater. Struct. 22055035

[61]. Collette C, Kroll G, Saive G, Guillemier V and Avraam M 2010 On magnetorheologic elastomers for vibration isolation, damping, and stress reduction in mass-varying structures J. Intell. Mater. Syst. Struct.21 1463-69

[62]. Deng H X and Gong X L 2007 Adaptive tuned vibration absorber based on magnetorheological elastomer J. Intell. Mater. Syst. Struct. 18 1205-10

[63]. Deng H X and Gong X L 2008 Application of magnetorheological elastomer to vibration absorber Commun. Nonlinear Sci. Numer. Simul. 13 1938-47

[64]. Lerner A A and Cunefare K A 2008 Performance of MRE-based vibration absorber J. Intell. Mater. Syst. Struct. 19 551-63.

[65]. Ni Z C, Gong X L, Li J F and Chen L 2009 Study on a dynamic stiffness-tuning absorber with squeezestrain enhanced magnetorheological elastomer J. Intell. Mater. Syst. Struct. 23 1195-202

[66]. Zhang X Z and Li W H 2009 Adaptive tuned dynamic vibration absorbers working with MR elastomers Smart Struct. Syst. 5 517-29

[67]. Xu Z, Gong X, Liao G and Chen X 2010 An active-damping-compensated magnetorheological elastomer adaptive tuned vibration absorber J. Intell. Mater. Syst. Struct.21 1039-47

[68]. Liao G J, Gong X L, Kang C J and Xuan S H 2011 The design of an active-adaptive tuned vibration absorber based on magnetorheological elastomer and its vibration attenuation performance Smart Mater. Struct. 20075015

[69]. Kim Y K, Koo J H, Kim K S, Kim S 2011 Developing a real time controlled adaptive MRE-based tunable vibration absorber system for a linear cryogenic cooler IEEE/ASME Int. Conf. Advanced Intelligent Mechatronics (Budapest, July 2011) pp 287-90

[70]. Sinko R, Karnes M, Koo J H, Kim K and Kim K S 2013 Design and test of an adaptive vibration absorber based on magnetorheological elastomers and a hybrid electromagnet J. Intell. Mater. Syst. Struct.24 803-12 
[71]. Sun S S, Chen Y, Yang J, Tian T F, Deng H X, Li W H, Du H and Alici G 2014 The development of an adaptive tuned magnetorheological elastomer absorber working in squeeze mode Smart Mater. Struct. 23 075009

[72]. Opie S and Yim W 2011 Design and control of a real-time variable modulus vibration isolator J. Intell. Mater. Syst. Struct. 22 113-25

[73]. Li W, Kostidis K, Zhang X and Zhou Y 2009 Development of a force sensor working with MR elastomers IEEE/ASME Int. Conf. Advanced Intelligent Mechatronics (Singapore, July 2009) pp 233-38

[74]. Yu M and Wang S 2010 The composite MRE embedded with a copper coil Smart Mater. Struct. 19 065023

[75]. Böse H, Rabindranath R and Ehrlich J 2012 Soft magnetorheological elastomers as new actuators for valves J. Intell. Mater. Syst. Struct. 23 989-94

[76]. Kavlicoglu B, Wallis B, Sahin H and Liu Y 2011 Magnetorheological elastomer mount for shock and vibration isolation Proc. SPIE 7977 Active and Passive Smart Structures and Integrated Systems 79770Y

[77]. Kashima S, Miyasaka F and Hirata K 2012 Novel soft actuator using magnetorheological elastomer IEEE Trans. Mag.48 1649-52

[78]. Li Y, Li J, Li W and Samali B 2013 Development and characterization of a magnetorheological elastomer based adaptive seismic isolator Smart Mater. Struct. 22035005.

[79]. Li J, Li Y, Li W and Samali B 2013 Development of adaptive seismic isolators for ultimate seismic protection of civil structures, Proc. SPIE 8692, Sensors and Smart Structures Technologies for Civil, Mechanical, and Aerospace Systems 2013, 86920H.

[80]. Fu J, Yu M, Dong X M and Zhu L X 2013 Magnetorheological elastomer and its application on impact buffer J. Phys.: Conf. Ser. 412012032

[81]. Wei K, Meng G and Hong Y 2009 Experimental study on vibration response behavior of a beam with magnetorheological elastomers J. Vib. Shock 28 81-83

[82]. Ying Z G and Ni Y Q 2009 Micro-vibration response of a stochastically excited sandwich beam with a magnetorheological elastomer core and mass Smart Mater. Struct. 18095005

[83]. Nayak B, Dwivedy S K and Murthy K S R K 2012 Multi-frequency excitation of magnetorheological elastomer-based sandwich beam with conductive skins Int. J. Non-Linear Mech. 47 448-60

[84]. Wei K X, Meng G, Zhang W M and Zhu S S 2008 Experimental investigation on vibration characteristics of sandwich beams with magnetorheological elastomers cores J. Central South Uni. Tech. 15 239-42

[85]. Hoang N, Zhang N and Du H 2009 A dynamic absorber with a soft magnetorheological elastomer for powertrain vibration suppression Smart Mater. Struct. 18074009

[86]. Hoang N, Zhang N and Du H 2011 An adaptive tunable vibration absorber using a new magnetorheological elastomer for vehicular powertrain transient vibration reduction Smart Mater. Struct. 20015019

[87]. Hoang N, Zhang N, Li W H and Du H 2013 Development of a torsional dynamic absorber using a magnetorheological elastomer for vibration reduction of a powertrain test rig J. Intell. Mater. Syst. Struct.24 2036-44

[88]. Eem S H, Jung H J and Koo J H 2011 Application of MR elastomers for improving seismic protection of base-isolated structures IEEE Trans. Mag.47 2901-04

[89]. Kim Y K, Bae H I, Koo J H, Kim K S and Kim S 2012 Real time control of a tunable vibration absorber based on magnetorheological elastomer for suppressing tonal vibrations Rev. Sci. Instrum. 83046108

[90]. Li W, Zhang X and Du H 2012 Development and simulation evaluation of a magnetorheological elastomer isolator for seat vibration control J. Intell. Mater. Syst. Struct.23 1041-48

[91]. Du H, Li W and Zhang N 2011 Semi-active variable stiffness vibration control of vehicle seat suspension using an MR elastomer isolator Smart Mater. Struct. 20105003

[92]. Jung H-J, Eem S-Hy, Jang D-D and Koo J-H 2011 Seismic Performance analysis of a smart baseisolation system considering dynamics of MR elastomer J. Intell. Mater. Syst. Struct. 22 1439-50.

[93]. Behrooz M, Wang X and Gordaninejad F 2014 Performance of a new magnetorheological elastomer isolation system Smart Mater. Struct. 23045014 
[94]. Zhou G Y and Wang Q 2005 Design of a smart piezoelectric actuator based on a magnetorheological elastomer Smart Mater. Struct. 14 504-510

[95]. Zhou G Y and Wang Q 2005 Magnetorheological elastomer-based smart sandwich beams with nonconductive skins Smart Mater. Struct. 14 1001-09

[96]. Zhou G Y and Wang Q 2006 Study on the adjustable rigidity of magnetorheological-elastomer-based sandwich beams Smart Mater. Struct. 15 59-74

[97]. Zhou G Y and Wang Q 2006 Use of magnetorheological elastomer in an adaptive sandwich beam with conductive skins. Part II: Dynamic properties - Discussion Int. J. Solids Struct., 43 5403-20.

[98]. Zhou G Y, Lin K C and Wang Q 2006 Finite element studies on field-dependent rigidities of sandwich beams with magnetorheological elastomer cores Smart Mater. Struct. 15 787-91

[99]. Dwivedy S K, Mahendra N and Sahu K C 2009 Parametric instability regions of a soft and magnetorheological elastomer cored sandwich beam J. Sound Vib. 325 686-704

[100]. Choi W J, Xiong Y P and Shenoi R A 2010 Vibration characteristics of sandwich beams with steel skins and magnetorheological elastomer cores Advances Struct. Eng. 13 837-47

[101]. Lara-Prieto V, Parkin R, Jackson M, Silberschmidt V and Kesy Z 2010 Vibration characteristics of MR cantilever sandwich beams: experimental study Smart Mater. Struct. 19015005

[102]. Miedzinska D, Boczkowska A and Zubko K 2010 Numerical verification of three point bending experiment of magnetorheological elastomer (MRE) in magnetic field J. Phys.: Conf. Ser. 240012158

[103]. Nayak B, Dwivedy S K and Murthy K S R K 2011 Dynamic analysis of magnetorheological elastomerbased sandwich beam with conductive skins under various boundary conditions J. Sound Vib. 330 183759

[104]. Korobko E V, Mikhasev G I, Novikova Z A and Zhurauski M A 2012 On damping vibrations of threelayered beam containing magnetorheological elastomer J. Intell. Mater. Syst. Struct. 23 1019-23

[105]. Yeh J Y 2013 Vibration analysis of sandwich rectangular plates with magnetorheological elastomer damping treatment Smart Mater. Struct. 22035010

[106]. Zhang X and Li W 2008 Research and application of MR elastomers Recent Patents on Mech. Eng. 1 161-66

[107]. Wang J and Meng G 2001 Magnetorheological fluid devices: principles, characteristics and applications in mechanical engineering Proc. Inst. Mech. Eng. L J. Mater. Des. Appl. 215 165-74

[108]. Goncalves F D and Carlson J D 2009 An alternate operation mode for MR fluids-magnetic gradient pinch J. Phys.: Conf. Ser. 149012050

[109]. Martin J E, Anderson R A, Odinek J, Adolf D and Williamson J 2003 Controlling percolation in fieldstructured particle composites: observations of giant thermoresistance, piezoresistance, and chemiresistance Phys. Rev. B 67094207

[110]. Zhu X, Jing X and Cheng L 2012 Magnetorheological fluid dampers: a review on structure design and analysis J. Intell. Mater. Syst. Struct. 23 839-73

[111]. Behrooz M, Wang X and Gordaninejad F 2014 Modeling of a new semi-active/passive magnetorheological elastomer isolator Smart Mater. Struct. 23045013

[112]. Gu X and Li Y 2013 Comprehensive investigation on magnetic field distribution in a solenoid The ASME 2013 Conference on Smart Materials, Adaptive Structures and Intelligent Systems, September 1618, 2013, Snowbird, Utah, USA. Paper ID: SMASIS 2013-3038. doi:10.1115/SMASIS2013-3038

[113]. Li Y, Li J and Samali B 2013 On the magnetic field and temperature monitoring of a solenoid coil for a novel magnetorheological elastomer base isolator J. Phys. Conf. Ser. 412012033

[114]. Liu K and Liu J 2005 The damped dynamic vibration absorbers: revisited and new result J Sound Vib. 284 1181-89

[115]. Sun H L, Zhang P Q, Gong X L and Chen H B 2007 A novel kind of active resonator absorber and the simulation on its control effect $J$ Sound Vib. 300 117-25

[116]. Ibrahim R A 2008 Recent advances in nonlinear passive vibration isolators J Sound Vib. 314 371-452

[117]. Jeong U C, Yoon J H, Yang I H, Jeong J E, Kim J S, Chung K H and Oh J E 2013 Magnetorheological elastomer with stiffness-variable characteristics based on induced current applied to differential mount of vehicles Smart Mater. Struct. 22115007 
[118]. Hwang I H, Lim J H and Lee J S 2006 A study on base isolation performance of magneto-sensitive rubbers J. Earthquake Eng. Soc. Korea 10 77-84.

[119]. Usman M, Sung S H, Jang D D, Jung H J and Koo J H 2009 Numerical investigation of smart base isolation system employing MR elastomer J. Phys. Conf. Ser. 149012009.

[120]. Martin J E 2005 Using traxial magnetic fields to create optimal particle composites Composites A 36 545-48

[121]. Martin J E, Anderson R A, Read D and Gulley G 2006 magnetostriction of field-structured magnetoelastomers Phys. Rev. E 74051507

[122]. Wang X, Gordaninejad F, Calgar M, Liu Y, Sutrisno J and Fuchs A 2009 Sensing behavior of magnetorheological elastomer J. Mech. Des. 131091004

[123]. Kchit N and Bossis G 2009 Electrical resistivity mechanism in magnetorheological elastomer Smart Mater. Struct. 42105505

[124]. Tian T F, Li W H and Deng Y M 2011 Sensing capacities of graphite based MR elastomers Smart Mater. Struct. 20025022

[125]. Bica I 2011 Magnetoresistor sensor with magnetorheological elastomers J Ind. Eng. Chem. 17 83-89

[126]. Du G T and Chen X D 2012 MEMS magnetometer based on magnetorheological elastomer Measurement 45 54-58

[127]. Yalcintas M and Dai H 2004 Vibration suppression capabilities of magnetorheological materials based adaptive structures Smart Mater. Struct.13 1-11

[128]. Hu G L, Guo M, Li W H, Du H P and Alici G 2011 Experimental investigation of the vibration characteristics of a magnetorheological elastomer sandwich beam under non-homogeneous small magnetic fields Smart Mater. Struct. 20127001

[129]. Hung H J, Lee S J, Jang D D, Kim I H, Koo J H and Khan F 2009 Dynamic characterization of magnetorheological elastomers in shear mode IEEE Trans. Magnetics 45 3930-33

[130]. Li W H, Yao G Z, Chen G, Yeo S H and Yap F F 2000 Testing and steady state modelling of a linear MR damper under sinusoidal loading Smart Mater Struc 9 95-102

[131]. Kamath GM and Wereley NM 1997 A nonlinear viscoelasticplastic model for electrorheological fluids Smart Mater Struc 6 351-59

[132]. D H Wang and W H Liao 2011 Magnetorheological fluid dampers: a review of parametric modelling Smart Mater. Struct. 20023001

[133]. Yang J, Du H, Li W, Li Y, Li J, Sun S and Deng H X 2013 Experimental study and modeling of a novel magnetorheological elastomer isolator Smart Mater. Struct. 22117001

[134]. Li Y and $\mathrm{Li} \mathrm{J} 2014$ A highly-adjustable base isolator utilizing magnetorheological elastomer: experimental testing and modeling ASME J. Vib. Acoustics doi: 10.1115/1.4027626

[135]. Li Y and Li J On rate-dependent mechanical models for high-damping magnetorheological elastomer base isolator Eng. Struct. under review

[136]. Yu Y, Li Y and Li J 2014 Parameter identification of a novel strain stiffening model for magnetorheological elastomer base isolator utilizing enhanced particle swarm optimization J. Intell. Mater. Syst. Struct. under review

[137]. Yu Y, Li Y and Li J 2014 A novel strain stiffening model for MRE isolator and parameter estimation using improved particle swarm optimization 6th World Conference on Structural Control and Monitoring, Barcelona, Spain, 11-17 July 2014.

[138]. Yu Y, Li Y and Li J 2014 Parameter identification of a new hysteretic model for magnetorheological elastomer base isolator using modified artificial fish swarm algorithm In Proc. of International Symposium on Automation and Robotics in Construction and Mining, Sydney, 2014, 9-11 July, paper ID: 196.

[139]. Yu Y, Li Y and Li J 2014 Parameter identification of an Improved Dahl model for magnetorheological elastomer base isolator based on enhanced genetic algorithm 23rd Australasian Conference on Mechanics of Structures and Materials (ACMSM23) Byron Bay, Australia, 9-12 December 2014.

[140]. Yu Y, Li Y and Li J 2014 Forecasting hysteresis behaviours of magnetorheological elastomer base isolator utilizing a hybrid model based on support vector regression and improved particle swarm optimization Sensor Sensor Actuat. A-Phys under review 
[141]. Li Y and Li J 2014 Finite element design and analysis of adaptive base isolator utilizing laminated multiple magnetorheological elastomer layers J. Intell. Mater. Syst. Struct. under review

[142]. Gu X, Li J, Askari M and Li Y 2014 Semi-active control of an innovative adaptive base isolation system employing magneto-rheological elastomer isolator J. Intell. Mater. Syst. Struct. under review

[143]. Gu X, Li J and Li Y 2014 Innovative semi-active storey isolation system using novel magnetorheological elastomer base isolators 23rd Australasian Conference on Mechanics of Structures and Materials (ACMSM23) Byron Bay, Australia, 9-12 December 2014.

[144]. Gu X, Li J and Li Y 2014 Adaptive base isolation system with magneto-rheological elastomer base isolators: numerical investigations, 6th World Conference on Structural Control and Monitoring, Barcelona, Spain, 11-17 July 2014. 